OPEN ACCESS

Edited by:

Yusuke Tsukamoto,

Kagoshima University, Japan

Reviewed by:

Kengo Tomida,

Osaka University, Japan Matthew Kunz,

Princeton University, United States

${ }^{*}$ Correspondence: James Wurster j.wurster@exeter.ac.uk

Specialty section This article was submitted to

Stellar and Solar Physics,

a section of the journal Frontiers in Astronomy and Space

Sciences

Received: 29 June 2018 Accepted: 15 November 2018 Published: 13 December 2018

Citation:

Wurster $J$ and Li Z-Y (2018) The Role of Magnetic Fields in the Formation of Protostellar Discs.

Front. Astron. Space Sci. 5:39 doi: 10.3389/fspas.2018.00039

\section{The Role of Magnetic Fields in the Formation of Protostellar Discs}

\author{
James Wurster ${ }^{1 *}$ and Zhi-Yun $\mathrm{Li}^{2}$ \\ ${ }^{1}$ School of Physics and Astronomy, University of Exeter, Exeter, United Kingdom, ${ }^{2}$ Astronomy Department, University of \\ Virginia, Charlottesville, VA, United States
}

The formation of a protostellar disc is a natural outcome during the star formation process. As gas in a molecular cloud core collapses under self-gravity, the angular momentum of the gas will slow its collapse on small scales and promote the formation of a protostellar disc. Although the angular momenta of dense star-forming cores remain to be fully characterized observationally, existing data indicates that typical cores have enough angular momenta to form relatively large, 100 au-scale, rotationally supported discs, as illustrated by hydrodynamic simulations. However, the molecular clouds are observed to be permeated by magnetic fields, which can in principle strongly affect the evolution of angular momentum during the core collapse through magnetic braking. Indeed, in the ideal magnetohydrodynamic (MHD) limit, magnetic braking has been shown to be so efficient as to remove essentially all of the angular momentum of the material close to the forming star such that disc formation is suppressed. This failure to produce discs in idealized cores is known as the magnetic braking catastrophe. The catastrophe must be averted in order for the all-important rotationally supported discs to appear, but when and how this happens remains debated. We review the resolutions proposed to date, with emphasis on misalignment, turbulence and especially non-ideal effects. Non-ideal MHD accounts for charged and neutral species, making it a natural extension to the ideal MHD approximation, since molecular clouds are only weakly ionized. The dissipative non-ideal effects diffuse the magnetic field to weaken it, and the dispersive term redirects the magnetic field to promote or hinder disc formation, dependent upon the magnetic geometry. When self-consistently applying non-ideal processes, rotationally supported discs of at least tens of au form, thus preventing the magnetic braking catastrophe. The non-ideal processes are sensitive to the magnetic field strength, cosmic ray ionization rate, and gas and dust grain properties, thus a complete understanding of the host molecular cloud is required. Therefore, the properties of the host molecular cloud - and especially its magnetic field - cannot be ignored when numerically modeling the formation and evolution of protostellar discs.

\section{Keywords: magnetic fields, magnetohydrodynamics (MHD), non-ideal MHD, star formation, protostellar discs}

\section{INTRODUCTION}

The broad outline of low-mass star formation has been known since at least (Larson, 1969), although many specific details are still under investigation. In Larson's description, which is the foundation for all current low-mass star formation models, a piece of the interstellar cloud (a molecular cloud core in modern terminology) collapses under self-gravity. The collapse is initially isothermal, 
since radiation is efficiently radiated away. However, as the density increases at the center of the core, it becomes optically thick to the radiation, which leads to an increase in thermal pressure support against self-gravity and the formation of the first hydrostatic or first Larson core. The first hydrostatic core continues to accrete material from the collapsing envelope, and its mass, density and temperature increase until the temperature rises above $\sim 2,000 \mathrm{~K}$; this temperature triggers the dissociation of $\mathrm{H}_{2}$, allowing the core to further collapse. This second collapse phase is rapid, and lasts until most of the $\mathrm{H}_{2}$ has been dissociated, at which point the second hydrostatic or stellar core has formed. The temperature continues to rise until nuclear burning starts and the star is formed.

The formation of star-forming molecular cloud cores is not fully understood ${ }^{1}$. These cores are observed to be initially slowly rotating, with ratios of rotational energy to gravitational potential being $\beta \lesssim 0.15$ with typical values of $\beta \sim 0.02$ (Goodman et al., 1993). However, their angular velocities are typically one to two orders of magnitude smaller than inferred by conservation of angular momentum (for a review, see Goldsmith and Arquilla, 1985). Therefore, there must exist some mechanism that will shed the angular momentum to allow these slowly rotating cloud cores to form (e.g., Spitzer, 1968).

As the rotating cloud core collapses under self-gravity, in the absence of magnetic fields, the rotation slows the collapse such that the gas forms a large protostellar disc as early as during the first core stage, and certainly by the Class 0 phase, as indicated by observations (e.g., Tobin et al., 2012; Murillo et al., 2013; Codella et al., 2014; Lee et al., 2017) and found in numerical simulations (e.g., Boss, 1993; Yorke et al., 1993, 1995; Boss and Myhill, 1995; Bate et al., 2014; Tomida, 2014; Wurster et al., 2018c). Conservation laws and observations thus both suggest that protostellar discs are a natural byproduct of the star formation process.

Molecular clouds are observed to be strongly magnetized (e.g., Crutcher, 1999; Bourke et al., 2001; Heiles and Crutcher, 2005; Troland and Crutcher, 2008), and magnetic fields are efficient at transporting angular momentum away from a collapsing core (known as "magnetic braking"; e.g., Mestel and Spitzer, 1956; Mouschovias and Paleologou, 1979, 1980; Basu and Mouschovias, 1994, 1995; Mellon and Li, 2008). On the cloud scale, magnetic braking likely occurs early in the cloud's formation and is responsible (at least in part) for reducing the angular momentum to the observed values (e.g., Mouschovias, 1983). Near the center of the collapsing core, magnetic braking means that discs are less necessary to conserve angular momentum since it is transported away. This reduced angular momentum may delay the formation of the disc until during or after the stellar core phase, or may prevent it altogether. In idealized numerical simulations including ideal magnetohydrodynamics (MHD), protostellar discs either fail to form or are much smaller than the observed sizes. This is known as the magnetic braking catastrophe (Allen et al., 2003; Galli et al., 2006).

\footnotetext{
${ }^{1}$ The focus of this review is on disc formation, thus for the remainder of this paper, we will assume that a slowly rotating cloud core has successfully formed.
}

Magnetic fields support charged gas against gravitational collapse, thus a common characterization of the relative importance of the gravitational and magnetic forces is the normalized mass-to-flux ratio,

$$
\mu \equiv \frac{M / \Phi_{\mathrm{B}}}{\left(M / \Phi_{\mathrm{B}}\right)_{\text {crit }}},
$$

where

$$
\frac{M}{\Phi_{\mathrm{B}}} \equiv \frac{M}{\pi R^{2} B},
$$

is the mass-to-flux ratio and

$$
\left(\frac{M}{\Phi_{\mathrm{B}}}\right)_{\mathrm{crit}}=\frac{c_{1}}{3 \pi} \sqrt{\frac{5}{G}},
$$

is the critical value where the gravitational and magnetic forces balance; in these equations, $M$ is the total mass contained within a core of radius $R, \Phi_{\mathrm{B}}$ is the magnetic flux threading the surface of the spherical core assuming a uniform magnetic field of strength $B, G$ is the gravitational constant and $c_{1} \simeq 0.53$ is a dimensionless coefficient numerically determined by Mouschovias and Spitzer (1976). The critical value of $\mu=1$ suggests that the gravitational and magnetic forces balance one another. For large super-critical values ( $\mu \gtrsim 20$ ), the magnetic field is inconsequential for core collapse, and the evolution is similar to that of a purely hydrodynamic cloud (e.g., Bate et al., 2014). For sub-critical values $(\mu<1)$, the magnetic field will prevent the collapse of the cloud core altogether. Observations suggest $\mu \sim 2-10$ in molecular cloud cores (e.g., Crutcher, 1999; Bourke et al., 2001; Heiles and Crutcher, 2005), however, this value could be even smaller after correcting for projection effects (Li et al., 2013a).

Although widely used, the mass-to-flux ratio should be used with caution, since the equation and the critical value are dependent on the geometry. While the above equations assume spherical geometry, a mass-to-flux ratio for a thin sheet is given in Nakano and Nakamura (1978), and the ratio for an oblate spheroid is given in Mouschovias and Spitzer (1976).

We will begin the review by describing the observational motivations in section 2, followed by a description of ideal MHD in the introduction to section 3. Our focus will then shift to numerical models, where we demonstrate the magnetic braking catastrophe (section 3.1), followed by attempts to prevent it while still keeping the ideal MHD approximation (sections 3.2 and 3.3). We will then introduce non-ideal MHD (section 4), and show the recent success of those simulations in preventing the magnetic braking catastrophe. We will conclude in section 5 .

\section{OBSERVATIONAL MOTIVATIONS}

The notion of a magnetized interstellar medium (ISM) dates back more than half a century, to at least the detection of polarized starlight (Hall, 1949; Hiltner, 1949) and its interpretation as coming from the absorption of the unpolarized starlight by magnetically aligned grains in the foreground medium (Davis and Greenstein, 1951; see Andersson et al., 2015 for a recent review). With the advent of observational capabilities, 
the magnetic fields in the ISM in general, and star-forming molecular clouds in particular, are becoming increasingly better characterized. For example, the PLANCK all-sky survey of the dust polarization leaves little doubt that a rather ordered magnetic field component exists in all nearby clouds (Planck Collaboration et al., 2015), as reviewed by H. B. Li in this volume. Observations have also revealed the prevalence of the magnetic field on the smaller scales of individual cores of molecular clouds and protostellar envelopes, as reviewed by Pattle et al., Crutcher \& Kemball, and Hull \& Zhang, in this volume.

As an illustration, we show in Figure 1 the dust polarization detected with the Atacama Large Millimeter/submillimeter Array (ALMA) around the Class 0 protostar B335 (Maury et al., 2018). The polarization orientations are rotated by $90^{\circ}$ to trace the magnetic field directions in the plane of the sky. It is immediately clear that not only a magnetic field is present on large scale, but also it shows coherent structures. In particular, the (projected) field appears to be significantly pinched near the equator of the system, as defined by the bipolar molecular outflows. The pinch is direct evidence that the magnetic field is interacting with the envelope material, through a magnetic tension force. Whether such a magnetic force is strong enough to affect the dynamics of the core collapse and especially disc formation is the question that we seek to address in this article.

There is some indirect evidence that magnetic fields may play a role in disk (and binary) formation. For example, Maury et al. (2010) concluded that core collapse models with a relatively strong magnetic field are more consistent with their IRAMPdBI observations of Class 0 protostellar systems than their hydrodynamic (non-magnetic) counterparts. In the particular case of B335, the specific angular momentum is observed to decrease rapidly toward the central protostar, with a rotationally supported disk (if present) smaller than $\sim 10$ au (Yen et al., 2015). The decrease in specific angular momentum and small disk could result naturally from the braking by a magnetic field, which has now been mapped in detail with ALMA (Maury et al., 2018). In addition, there is some tentative evidence that protostellar sources with misaligned magnetic field and rotation axis (inferred from outflow direction) tend to have larger disks (e.g., Segura-Cox et al., 2016), which is consistent with magnetized disk formation simulations (e.g., Hennebelle and Ciardi, 2009; Joos et al., 2012; Krumholz et al., 2013; Li et al., 2013b).

Finding evidence for the magnetic field on the disc scale is more challenging. Spatially resolved dust polarization has been detected in discs around a number of young stellar objects, using the Submillimeter Array (SMA; e.g., Rao et al., 2014), the Combined Array for Research in Millimeter-wave Astronomy (CARMA; e.g., Stephens et al., 2014; Segura-Cox et al., 2015), the Very Large Array (VLA; e.g., Cox et al., 2015; Liu et al., 2016), and especially ALMA (e.g., Kataoka et al., 2017; Stephens et al., 2017; Alves et al., 2018; Bacciotti et al., 2018; Cox et al., 2018; Girart et al., 2018; Harris et al., 2018; Hull et al., 2018; Lee et al., 2018; Sadavoy et al., 2018; Dent et al., 2019). However, with the exception of BHB 07-11 (Alves et al., 2018) and possibly a few other cases, the majority of the sources do not show any evidence for dust grains aligned by the generally expected toroidal magnetic fields; their polarization patterns are better explained by dust scattering instead (Kataoka et al., 2015, 2016; Yang et al., 2016a,b, 2017). The reader is referred to Hull \& Zhang's article in this volume for a more detailed discussion. In any case, whether and how the disc is connected to the protostellar envelope through a magnetic field remain to be determined observationally.

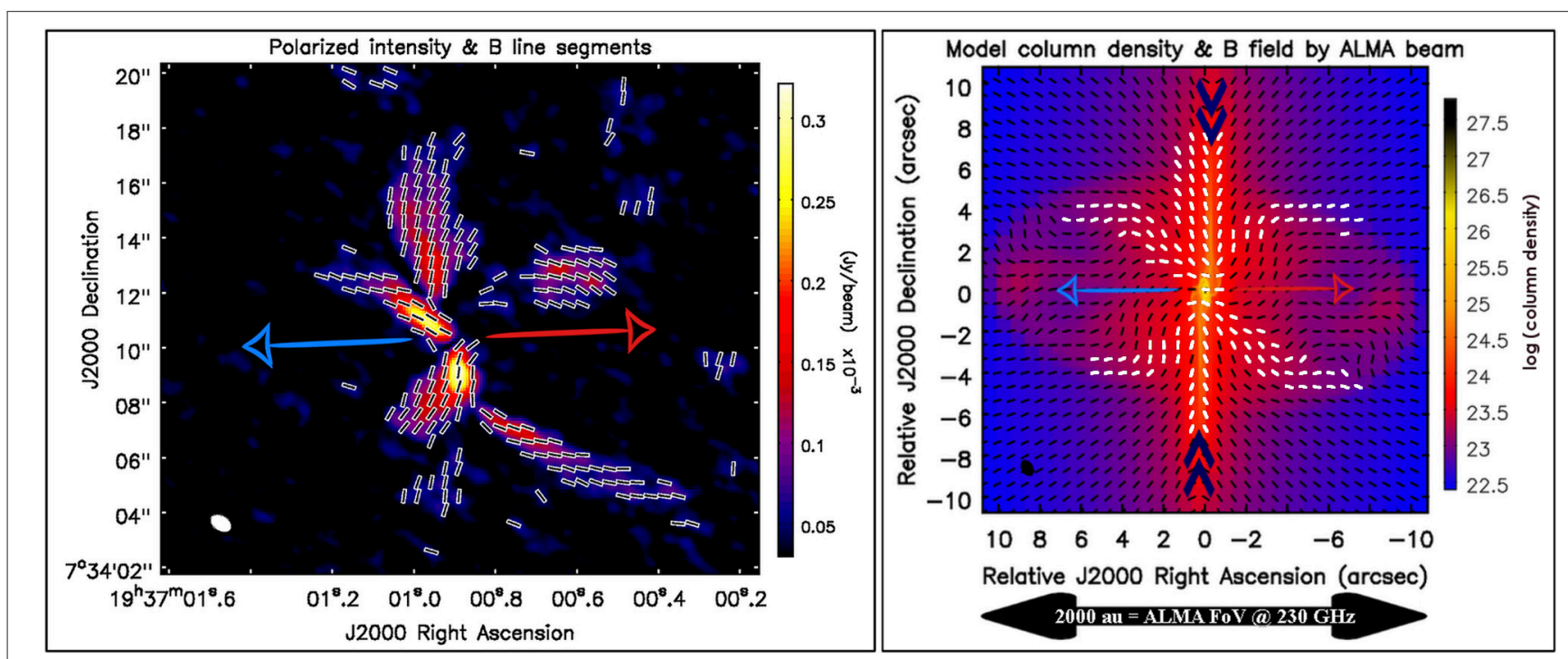

FIGURE 1 | An example of the magnetic field traced by dust polarization around an observation (left-hand panel) and a numerical model (right-hand panel) of the solar-type Class 0 protostar B335. The background image in the right-hand panel is the ALMA polarized dust continuum emission, and the superimposed lines infer the magnetic field orientations (i.e. the polarization angle rotated by $90^{\circ}$ ). This figure is inspired by Figures 1 and 3 of Maury et al. (2018), and was created by A. J. Maury for this publication. 


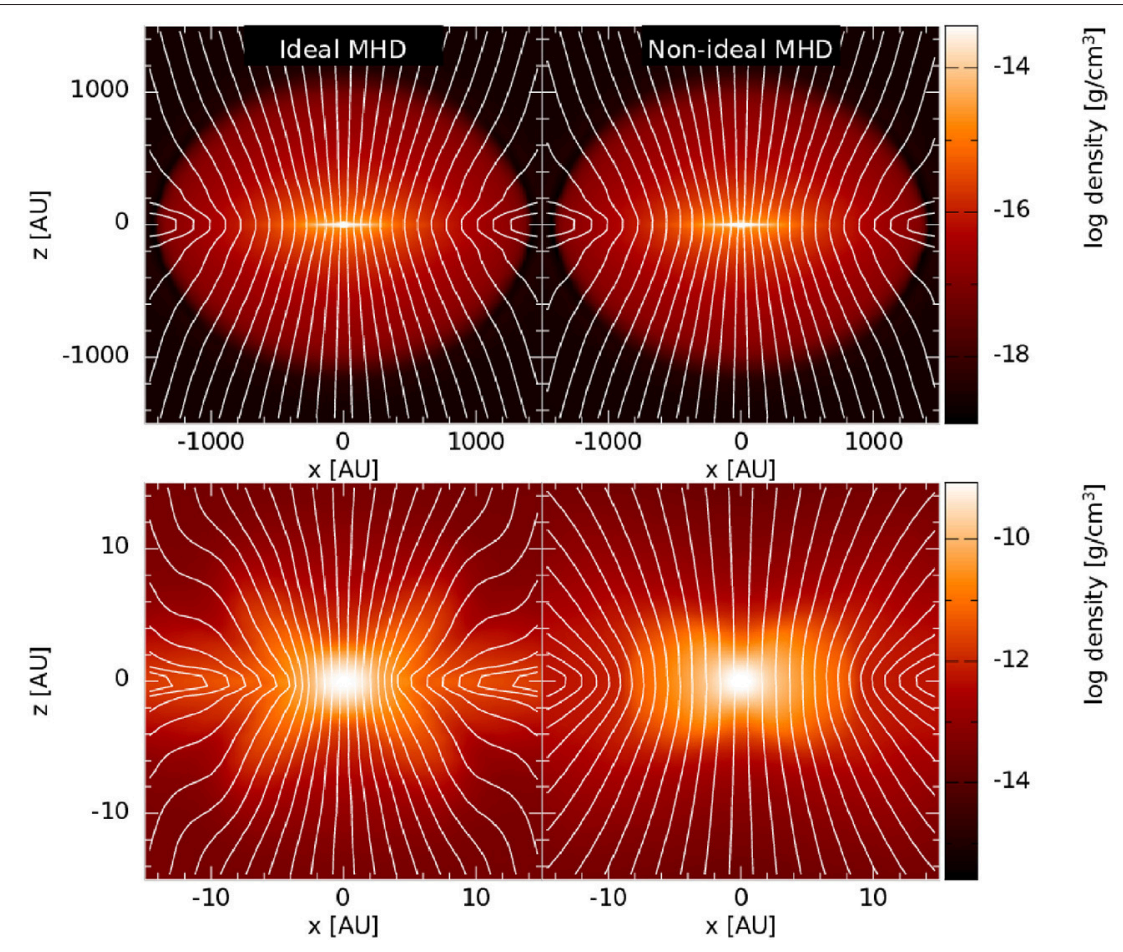

FIGURE 2 | The magnetic field lines superimposed on a density slice during the first hydrostatic core phase for ideal and non-ideal MHD simulations. The initial mass-to-flux ratio is five times the critical value (i.e., $\mu_{0}=5$ ). In ideal MHD, the magnetic field lines are dragged inwards as the cloud core collapses, creating the characteristic hour-glass shape. On the large scale, the non-ideal effects have minimal effect on the strength and structure of the magnetic field, whereas on the small scale, the neutral particles flow through the magnetic field lines to form the first hydrostatic core, while preventing the magnetic field lines from becoming pinched and preventing a strong magnetic field from building up in the core (bottom). These images are inspired by Figure 2 of Price and Bate (2007) and Figure 1 of Bate et al. (2014).

\section{DISC FORMATION IN THE IDEAL MHD LIMIT}

The simplest approximation when modeling magnetic fields is to use ideal MHD, where it is assumed that the gas is sufficiently ionized such that the magnetic field is well coupled to the bulk neutral gas. In this approximation, the induction equation is given by

$$
\frac{\partial \boldsymbol{B}}{\partial t}=\nabla \times(\boldsymbol{v} \times \boldsymbol{B})
$$

where $\boldsymbol{v}$ is the gas velocity and $\boldsymbol{B}$ is the magnetic field. Since the gas is tied to the magnetic field lines, the lines are dragged in as the gas collapses (assuming $\mu_{0}>1$ ), causing a characteristic hour-glass shape; see the left-hand column of Figure 2 for numerical results, which nicely complement the observational results in Figure 1. This pinching effect becomes less prominent as the magnetic field becomes stronger, since the stronger field is harder to bend (see Figure 2 of Price and Bate, 2007). If the magnetic field were to be dragged all the way into the central stellar object, then the stellar field strength would be millions of gauss, which is much higher than the kilo-gauss field typically observed in young stars. This is a manifestation of the socalled "magnetic flux problem" in star formation (Babcock and Cowling, 1953; Mestel and Spitzer, 1956; Shu et al., 2006)².

In purely hydrodynamics simulations, large protostellar discs can form due to conservation of angular momentum. In the presence of magnetic fields, angular momentum can be efficiently transported away from the collapsing central region (e.g., Mestel and Spitzer, 1956; Mouschovias and Paleologou, 1979, 1980; Basu and Mouschovias, 1994, 1995; Mellon and Li, 2008), and not enough angular momentum remains for a rotationally supported disc to form. This is the magnetic braking catastrophe, as first demonstrated by Allen et al. (2003, see also the pioneering work by Tomisaka, 2000): Rotationally supported discs do not form in idealized numerical simulations in the presence of magnetic fields of realistic strengths. Analytical studies by Joos et al. (2012) estimated that $\mu \leq 10$ should be enough to suppress disc formation.

There have been many numerical simulations of disc formation under the assumption of ideal MHD. Most simulations are initialized with a rotating spherical cloud core which is threaded with a magnetic field that is parallel to the rotation axis (section 3.1). However, molecular clouds contain turbulent flows

${ }^{2}$ Given the focus of this review, the magnetic flux problem will not be addressed here; see Wurster et al. (2018d) for a recent discussion. 
(e.g., Heyer and Brunt, 2004), which form large scale structures (e.g., Padoan and Nordlund, 2002; McKee and Ostriker, 2007; Ward-Thompson et al., 2010), and it is these chaotic structures that can collapse to form cores that ultimately collapse to form stars and protostellar discs. Thus, a more realistic scenario is that the magnetic fields are initially misaligned with the rotation axis (see section 3.2 ), or the velocity field initially contains a significant turbulent component (see section 3.3).

\subsection{Idealized Initial Conditions}

The simplest and most common initial condition for disc formation from a collapsing molecular cloud core is to thread a magnetic field parallel to the rotation axis of a spherical core that is in solid-body rotation; the initial magnetic field strength is characterized by the initial mass-to-flux ratio, $\mu_{0}$, for the core as a whole. The early ideal MHD simulations were performed under the assumption of an isothermal or barotropic equation of state and were performed in two-dimensional (e.g., Allen et al., 2003; Mellon and Li, 2008) or three-dimensional (e.g., Machida et al., 2004; Price and Bate, 2007; Hennebelle and Fromang, 2008; Duffin and Pudritz, 2009; Machida et al., 2011; Zhao et al., 2011; Santos-Lima et al., 2012; Seifried et al., 2012). Later studies were radiative three-dimensional calculations that included simplified ideal magnetic fields calculations (e.g., Boss, 1997, 1999), or solved the complete MHD equations (e.g., Boss, 2002, 2005, 2007, 2009; Commerçon et al., 2010; Tomida et al., 2010, 2013; Bate et al., 2014). Subsequent studies included radiation and ideal magnetic fields as part of a parameter study (e.g., Tomida, 2014; Tomida et al., 2015; Tsukamoto et al., 2015b; Vaytet et al., 2018; Wurster et al., 2018a,c,d). When using moderate to strong magnetic fields, these studies all found efficient magnetic braking, and none of them formed a protostellar disc.

For a demonstration of the magnetic braking catastrophe, Bate et al. (2014) simulated four magnetized models and one hydrodynamical model. Figure 3 shows the face-on and edge-on gas densities in a slice through the first hydrostatic core, and these figures are representative of ideal MHD models in the literature. With weak or no magnetic fields ( $\mu_{0}=100$, Hydro), the first core is rotating quickly enough and is massive enough to become bar unstable and forms a gravitationally unstable disc that is dominated by spiral arms (e.g., Bate, 1998, 2010, 2011; Saigo and Tomisaka, 2006; Saigo et al., 2008; Machida et al., 2010).

As analytically predicted by Joos et al. (2012), there are no discs in the models with $\mu_{0} \leq 10$, however, pseudo-discs do form; a pseudo-disc is an over-density of gas around a protostar that is not centrifugally supported, not in equilibrium, and is resulted from the anisotropy of the magnetic support against gravity (Galli and Shu, 1993; Li and Shu, 1996), although, throughout the literature, authors use this term to refer to a variety of disc-like structures. The pseudo-discs in Bate et al. (2014) do not increase in size, nor do they ever become Keplerian discs. This study clearly demonstrates the magnetic braking catastrophe, at least up to the formation of the first hydrostatic core.

When considering the long term evolution of the system, discs may yet form. In their ideal MHD simulations, Machida and Hosokawa (2013) find that discs form in their models by the end of the Class 0 phase, and increase in mass into the Class I phase.
As the envelope is depleted, the magnetic braking becomes less efficient, which allows these discs to form as speculated earlier by Mellon and Li (2008, see also section 4.2.1); when their strongly magnetized models end in the Class I phase, the discs have masses $\sim 40$ per cent of the mass of the protostar itself.

This leads to the open question of when protostellar discs form. If they form in later stages (e.g., Class I or II), then there may be no magnetic braking catastrophe in the numerical simulations; if they form early in the Class 0 phase, then the catastrophe persists, and one must go beyond the idealized initial conditions to form a discs if the magnetic field is strong and wellcoupled to the gas. Future observations are required to determine when in the star formation process its protostellar disc forms.

\subsection{Misaligned Magnetic Fields}

There have been several studies investigating the impact of misaligned magnetic fields on the formation of discs (eg., Matsumoto and Tomisaka, 2004; Machida et al., 2006; Matsumoto et al., 2006; Hennebelle and Ciardi, 2009; Joos et al., 2012; Krumholz et al., 2013; Li et al., 2013b; Lewis et al., 2015; Lewis and Bate, 2017). Similar to the literature, we define the angle $\theta$ such that the angular momentum $\boldsymbol{J}$ and magnetic field $\boldsymbol{B}$ vectors are parallel and aligned when $\theta \equiv 0^{\circ}$. The components of the angular momentum that are parallel and perpendicular to the magnetic field are $\boldsymbol{J}_{\|}=|\boldsymbol{J} \cdot \boldsymbol{B}| /|\boldsymbol{B}|$ and $\boldsymbol{J}_{\perp}=|\boldsymbol{J} \times \boldsymbol{B}| /|\boldsymbol{B}|$, respectively.

Two-dimensional analytical models of collapsing cylinders by Mouschovias and Paleologou (1979) found that magnetic braking can reduce the angular momentum of a cloud by a few orders of magnitude if $\theta=90^{\circ}$. All other parameters being the same, this indicates that systems with $\theta=0^{\circ}$ are more likely to form discs than their $\theta=90^{\circ}$ counterparts. However, this pioneering work did not include the gravitational collapse, which can modify the magnetic field configuration and affect the braking efficiency.

The results of Mouschovias and Paleologou (1979) were later confirmed by the three-dimensional models of Matsumoto and Tomisaka (2004). In these models, the perpendicular component of the angular momentum, $J_{\perp}$, decreased faster than the parallel component, indicating that magnetic braking was more efficient for the perpendicular component. This component decreased rapidly and by a few orders of magnitude in their models with $\theta=45$ and $90^{\circ}$; the component $\boldsymbol{J}_{\|}$decreased only by a factor of a few in their models with $\theta=0$ and $45^{\circ}$ (see their Figure 4 ). These results broadly agree with the parameter study by Machida et al. (2006), who also find that magnetic braking acts primarily on the component perpendicular to the rotation axis. They conclude that discs form more easily when $\theta=0^{\circ}$.

Several studies, however, reach the opposite conclusion: Discs form more easily when $\theta=90^{\circ}$. Joos et al. (2012) find that massive discs form in all of their misaligned models, requiring as little as $\theta=20^{\circ}$ to allow a massive disc to form. The exceptions are their models with the strongest magnetic field strength, $\mu_{0}=$ 2 , in which discs never form, independent of $\theta$. As the evolution progresses, the pseudo-discs continue to accrete, increasing both their mass and angular momentum; more massive discs form for larger $\theta$, and faster rotating discs form for weaker magnetic fields (larger $\left.\mu_{0}\right)$. 


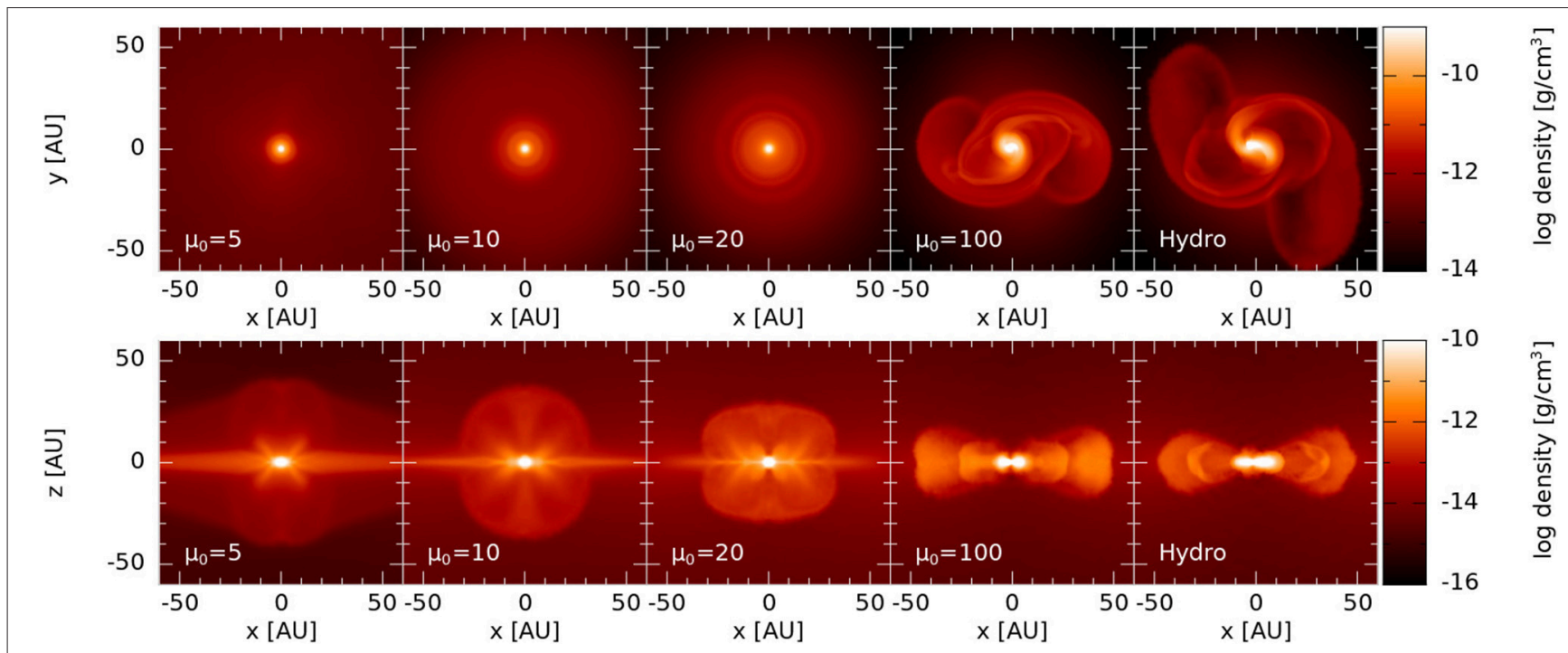

FIGURE 3 | The face-on (top row) and edge-on (bottom row) gas density in a slice through the centre of the first hydrostatic core for ideal MHD models of decreasing magnetic field strength (increasing $\mu_{0}$; left to right) and a pure hydrodynamics model. Gravitationally unstable discs form for $\mu_{0}>20$, whereas only pseudo-discs only form in the remaining models. This is inspired by Figure 4 of Bate et al. (2014), and was created for this publication using the data from Bate et al. (2014).

Except in the case of very strong magnetic fields, Li et al. (2013b) find the initially misaligned magnetic field allows rotationally supported discs to form in the dense cores, even when no discs form in the aligned models. In their models, the magnetic field lines are wrapped into a snail-shaped curtain when $\theta=90^{\circ}$, and this configuration hinders outflows. With negligible outflows, the angular momentum remains near the protostar, allowing the formation of the disc.

In Lewis and Bate (2017), the pseudo-disc increases in size and forms larger arms as the misalignment increases since the gas can easily flow along the horizontal magnetic field component. For $\theta=20$ and $45^{\circ}$, the pseudo-discs are warped such that the inner regions are perpendicular to the rotation-axis, while the outer regions are perpendicular to the magnetic field.

In summary, misalignment between the rotation axis and the magnetic field lines may promote or hinder the formation of rotationally supported discs. Machida et al. (2006) found that for slow rotators, magnetic braking aligns the rotation axis and the magnetic field (in agreement with Lewis and Bate, 2017 if comparing the outer parts of the discs), and for fast rotators, the magnetic field aligns through a dynamo action. This suggests that the effect of misalignment may, at least in part, be a result of the initial conditions. Thus, at the time of writing, the effect of misalignment on disc formation is inconclusive.

\subsection{Turbulent Initial Conditions}

There are several studies of disc formation in massive turbulent magnetized molecular clouds $\left(M>100 \mathrm{M}_{\odot}\right.$; e.g., Santos-Lima et al., 2012, 2013; Seifried et al., 2012, 2013; Myers et al., 2013; Li et al., 2014; Fielding et al., 2015; Gray et al., 2018), as well a number of studies that begin from turbulent, low-mass cores ( $M<10 \mathrm{M}_{\odot}$; e.g., Matsumoto and Hanawa, 2011; Joos et al., 2013; Li et al., 2014; Matsumoto et al., 2017; Lewis and Bate,
2018); for scales consistent with this review, we focus on the latter studies. These low-mass simulations reach contradicting results, with some studies suggesting increased turbulence promotes disc formation (Joos et al., 2013; Li et al., 2014), while others suggest it hinders discs formation (Matsumoto and Hanawa, 2011; Matsumoto et al., 2017; Lewis and Bate, 2018).

Figure 4 illustrates the effect of how increasing the Mach number, $\mathcal{M}$, of the turbulent velocity field imposed on a slowly rotating, pre-stellar cloud core hinders disc formation. As the Mach number is increased, the resulting pseudo-disc is smaller, and the rotating gas becomes less Keplerian. For $\mathcal{M}=1$, the system is disrupted and no pseudo-disc forms. In the most turbulent model, the initial ratio of turbulent to rotational energy is $E_{\text {turb }} / E_{\text {rot }}=26$, and Lewis and Bate (2018) argue that $E_{\text {turb }} / E_{\text {rot }} \lesssim 1$ is required for the formation of a pseudo-disc. By increasing the initial rotation such that $E_{\text {turb }} / E_{\text {rot }}=1.6$, they form a disrupted pseudo-disc, while increasing it such that $E_{\text {turb }} / E_{\text {rot }}=1.06$, they form a slowly rotating pseudo-disc.

On slightly larger spatial scales before the first hydrostatic core forms, Matsumoto and Hanawa (2011) find that models without turbulence produce axisymmetric oblate or prolate clouds (depending on initial mass). As the turbulence is increased, the clouds become more chaotic and disrupted. As the gravitational collapse continues, each model eventually forms a spherical first core surrounded by a disc-like envelope (with the exception of one model with weak magnetic fields and moderate turbulence).

Following the long-term evolution of their turbulent models, Matsumoto et al. (2017) formed a disc in each model, and the disc mass and radius increased with time. In agreement with non-turbulent studies, they consistently found larger discs in models with weaker magnetic fields. However, they also consistently found larger discs in models with weaker turbulence (all other parameters being held constant). Thus, they concluded, turbulence hindered disc formation. 


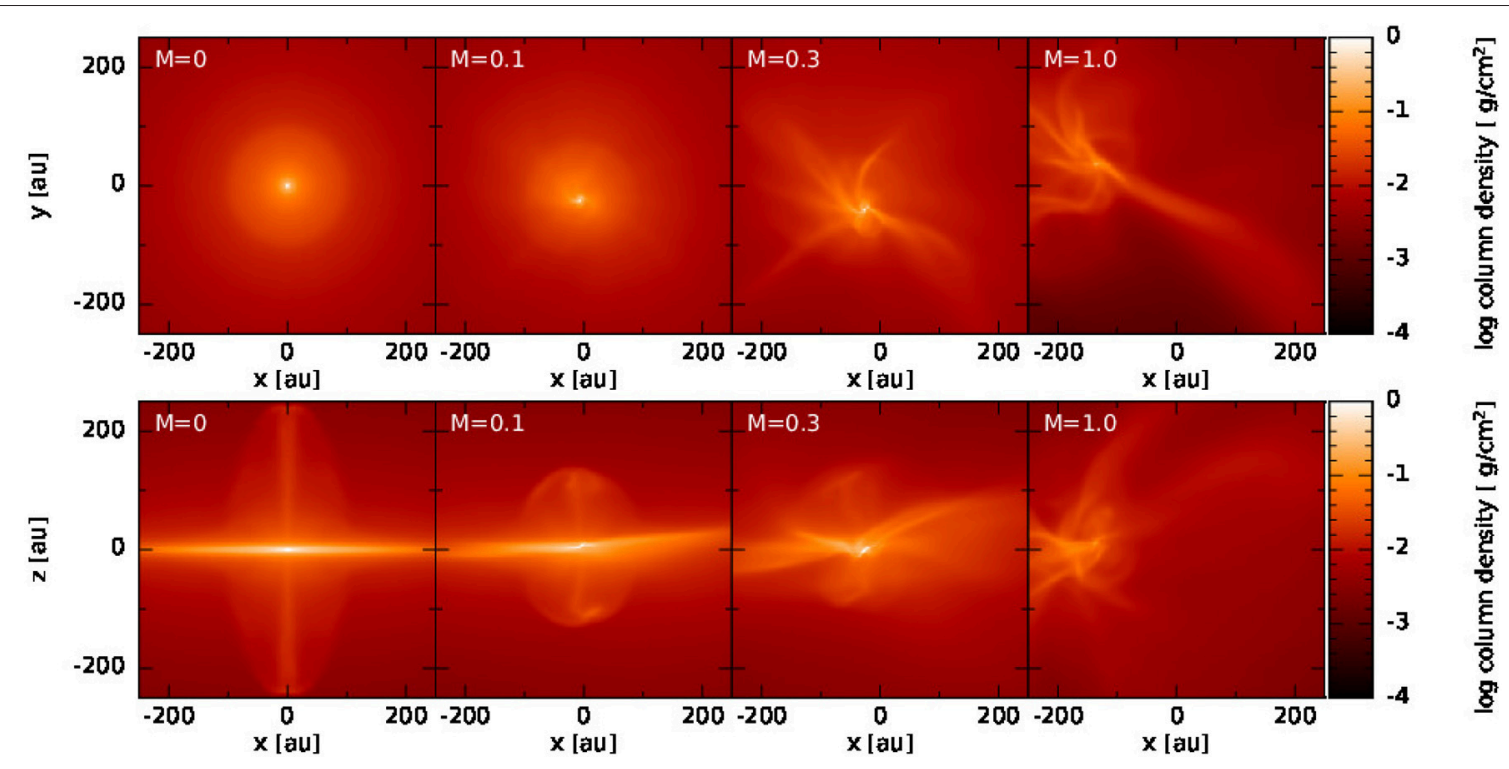

FIGURE 4 | The face-on (Top row) and edge-on (Bottom row) gas column density projections showing disc formation in models in a relatively strong ( $\mu_{0}=5$ ) magnetic field with a turbulent velocity field imposed onto a solid-body rotation. The Mach number is shown in each panel. The figures are taken $\sim 500$ yr after sink formation (i.e., when the maximum density has reached $\rho$ max $=10^{-10} \mathrm{~g} \mathrm{~cm}^{-3}$ ). Increasing turbulence in these models hinders disc formation. These are inspired by Figure 7 of Lewis and Bate (2018), but are from lower resolution models.

In their strongest magnetic field model, the disc radii and masses were nearly indistinguishable between their two turbulent models $(\mathcal{M}=0.5,1$; see their Figures 5, 6 ), suggesting that at these magnetic field strengths, the strength of turbulence played a secondary role in the cloud's evolution.

Contrary to the above, Figure 5 illustrates the effect of how increasing the Mach number, $\mathcal{M}$ promotes disc formation. In the models of Li et al. (2014), a disc-like structure begins to form at $\mathcal{M}=0.5$, however, it is still partially disrupted. At larger Mach numbers, the disc becomes more prominent, and for $\mathcal{M}=1$, has a Keplerian rotational profile. They conclude that the promotion of disc formation is a result of the warping of the pseudo-disc and the magnetic decouplingtriggered reconnection of the severely pinched field lines near the central object.

Joos et al. (2013) presented a suite of models, and found massive discs in all their simulations with weak magnetic fields, and very small discs in their strongly magnetized models; at both strong and weak magnetic field strength, the disc growth rate is approximately independent of the Mach number. For moderate magnetic field strengths, the disc growth rates are dependent on the Mach number such that a $\sim 0.6 \mathrm{M}_{\odot}$ disc forms in the same length of time it takes to form a $\sim 0.4 \mathrm{M}_{\odot}$ disc in a laminar model (the model has an initial mass of $5 \mathrm{M}_{\odot}$ ). In these models, the turbulence diffuses the magnetic field out of the central region, generating an effective magnetic diffusivity prompted by magnetic reconnection, and hence weakening the magnetic field (see also Weiss, 1966; Santos-Lima et al., 2012). Turbulence also induces a misalignment between the rotation axis and the magnetic field (see also Seifried et al., 2012; Gray et al., 2018) of $20-60^{\circ}$, which reduces the magnetic braking. The effect of these two mechanisms is to allow for larger discs to form.

In summary, turbulence can hinder or promote disc formation. Thus, as with the studies of initial magnetic field alignment, initial conditions will likely play an important role in determining the outcome.

\section{NON-IDEAL MHD AND DISC FORMATION}

It is well known that the dense, star-forming, cores of molecular clouds are lightly ionized (Bergin and Tafalla, 2007), with detailed models finding ionization fractions as low as $n_{\mathrm{e}} / n_{\mathrm{H}_{2}}=10^{-14}$ (Nakano and Umebayashi, 1986; Umebayashi and Nakano, 1990; Nishi et al., 1991; Nakano et al., 2002). The low ionization level means that the magnetic field is no longer perfectly coupled to the bulk neutral material, rendering the ideal MHD approximation questionable. A proper treatment of the nonideal MHD effects is required, including a detailed calculation of the abundances of the electrons, ions and charged dust grains.

There are several methods of numerically modeling multiple species. They can be modeled explicitly (e.g., Inoue et al., 2007; Inoue and Inutsuka, 2008, 2009), where each species has its own continuity and momentum equation. In this method, the species interact directly with each other through terms in the momentum and energy equations. Electrons are not explicitly treated because their mass is much less than those of other particles. The induction equation is as given in Equation 4, except that it only includes the velocity of the charged species. 


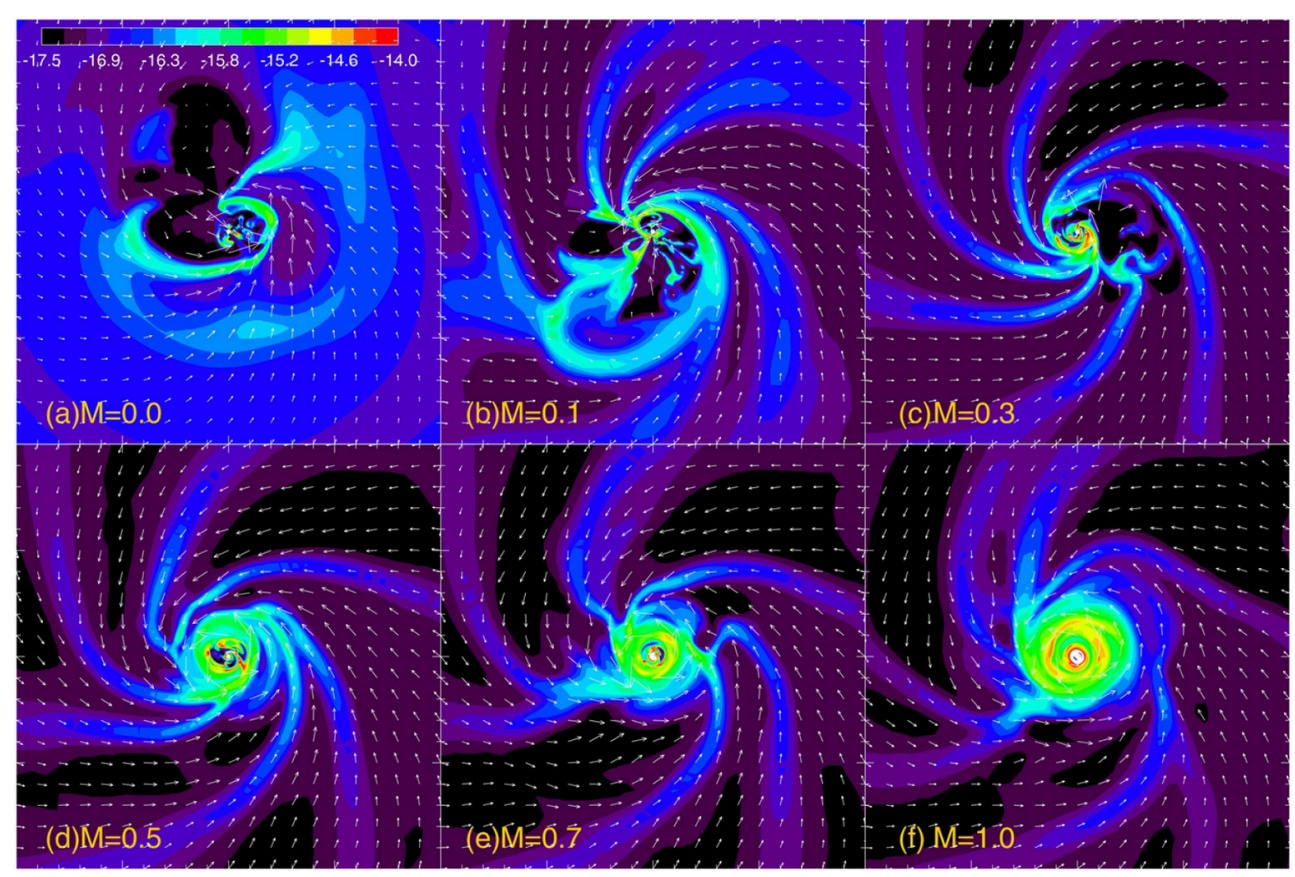

FIGURE 5 | The gas density (color) and velocity (vectors) in the mid-plane for models with increasing Mach number (printed in the bottom left-hand corner of each frame). Each frame is $\sim 1,400$ au on each side. In this suite of simulations, increasing the Mach number promotes disc formation. This is Figure 2 of Li et al. (2014). ${ }^{(\odot)}$ AAS. Reproduced with permission.

Under the assumptions that mass density is dominated by the neutral mass density, and that collisions occur predominantly between charged species and neutrals, then the inertia and pressure of the charged species and collisions between charged species can be safely neglected (e.g., O'Sullivan and Downes, 2006; Rodgers-Lee et al., 2016). In this approximation, each species has its own continuity equation, but only the neutral species has a momentum equation; additional equations are included to govern the interactions (e.g., energy transfer) between the species. Other studies include dust grains, and include continuity equations for the neutral gas and total grain density but not the charged gas species (e.g., Ciolek and Mouschovias, 1993, 1994; Tassis and Mouschovias, 2005a,b, 2007a,b,c; Kunz and Mouschovias, 2009, 2010).

The continuity equation can be constructed to evolve total mass density rather than species mass density if the strong coupling approximation is invoked; in this approximation, the ion pressure and momentum are negligible compared to that of the neutrals, and the magnetic field and neutral flows evolve on a timescale that is long compared to the timescale of the charged particles (e.g., Wardle and Koenigl, 1993; Ciolek and Mouschovias, 1994; Mac Low et al., 1995; Wardle and Ng, 1999; Choi et al., 2009). In approximation, there is one continuity and one momentum equation. This method is typically used by those studying the formation of protostellar discs (e.g., Krasnopolsky et al., 2010; Tomida et al., 2015; Tsukamoto et al., 2015b,a; Masson et al., 2016; Wurster et al., 2016; Vaytet et al., 2018; Wurster et al., 2018c).
Except in the cases where the charged and neutral species interact directly through their own momentum equations, the induction equation is modified to account for the species of different charges, viz.,

$$
\begin{aligned}
\frac{\partial \boldsymbol{B}}{\partial t} & =\left.\frac{\partial \boldsymbol{B}}{\partial t}\right|_{\text {ideal }}+\left.\frac{\partial \boldsymbol{B}}{\partial t}\right|_{\text {non-ideal }} \\
& =\nabla \times(\boldsymbol{v} \times \boldsymbol{B})-\nabla \times \\
& \left\{\eta_{\mathrm{O}}(\nabla \times \boldsymbol{B})+\eta_{\mathrm{H}}(\nabla \times \boldsymbol{B}) \times \hat{\boldsymbol{B}}-\eta_{\mathrm{A}}[(\nabla \times \boldsymbol{B}) \times \hat{\boldsymbol{B}}] \times \hat{\boldsymbol{B}}\right\},
\end{aligned}
$$

where all the micro-physics governing the species properties and interactions is contained within the coefficients, $\eta$. In the case of, e.g., O'Sullivan and Downes (2006) and Rodgers-Lee et al. (2016), the species densities are taken directly from the continuity equation, whereas sub-grid algorithms are required if not explicitly evolving the density of the charged species or if using the strong coupling approximation. The three coefficients represent the different regimes of interactions between the neutrals and the charged particles:

1. Ohmic resistivity, $\eta_{\mathrm{O}}$ : ions, electrons and charged grains are completely decoupled from the magnetic field,

2. Hall effect (ion-electron drift), $\eta_{\mathrm{H}}$ : massive particles (ions, charged grains) are decoupled from the magnetic field while electrons remain coupled (i.e., the electrons are frozen into the magnetic field, which drifts through the ions and charged grains), and 
3. Ambipolar diffusion (ion-neutral drift), $\eta_{\mathrm{A}}$ : both massive charged particles (ions, charged grains) and electrons are coupled to the magnetic field (i.e., the charged particles are frozen into the magnetic field, which drifts through the neutrals).

Ohmic resistivity and ambipolar diffusion are both diffusive terms, with the associated energy dissipation given by Wurster et al. (2014)

$$
\frac{\partial u}{\partial t}=\left.\frac{\partial u}{\partial t}\right|_{\text {ideal }}+\frac{\left|\nabla \times \boldsymbol{B}^{2}\right|}{\rho} \eta_{\mathrm{O}}+\frac{1}{\rho}\left\{|\nabla \times \boldsymbol{B}|^{2}-[(\boldsymbol{\nabla} \times \boldsymbol{B}) \cdot \hat{\boldsymbol{B}}]^{2}\right\} \eta_{\mathrm{A}},
$$

where $u$ is the internal energy. Physically, these non-ideal processes allow the neutral and selected ionized particles to slip through the magnetic field, which typically leads to a redistribution of the magnetic field lines relative to the bulk neutral matter. In particular, a concentration of matter may not lead to as large an increase in the magnetic field strength as in the ideal MHD limit.

Ohmic resistivity is typically important at the highest densities, such as the inner midplane regions of the protostellar disc, while ambipolar diffusion typically dominates at relatively low densities, such as the molecular cloud core itself, and the upper and outer regions of the protostellar disc (e.g., Shu et al., 2006; Wardle, 2007; Machida et al., 2008; Wurster et al., 2018a). Figure 2 shows the magnetic field lines on both the molecular cloud core and protostellar disc scales for ideal and non-ideal MHD. When using ideal MHD, the magnetic field lines are dragged into the center, causing an enhancement in the magnetic field strength, and creating the expected "hour-glass shaped" field lines. Since the neutral gas can slip through the magnetic field lines in the non-ideal MHD case, the field lines do not become as pinched, resulting in a weaker central magnetic field strength.

The Hall effect is dispersive rather than dissipative, and typically dominates at the intermediate densities between the two diffusive regimes, including in parts of the dense core and protostellar disc (e.g.) Sano and Stone, 2002a,b; see Figure 3 of Li et al., 2011 for an illustrative example). Unlike the other terms, the Hall effect can change the magnetic geometry of the system without any dissipation. Specifically, it will generate a toroidal magnetic field from a poloidal magnetic field, where the resulting magnetic torques can induce a rotation in an otherwise nonrotating medium (for an example, see Figure 15 of Li et al., 2011; for a sketch of the processes, see Figure 1 of Tsukamoto et al., 2017). When the polarity of the initial poloidal magnetic field is reversed, the resulting toroidal magnetic field and rotation would also be in the opposite direction. This has implications if the system is already rotating, since the Hall effect will either transport angular momentum to or from the central region, thus will either increase or decrease the angular velocity of the local gas (Wardle, 2007; Braiding and Wardle, 2012a). Assuming $\eta_{\mathrm{H}}<0$ (as is reasonable in protostellar discs; Tsukamoto et al., 2015a; Marchand et al., 2016; Wurster, 2016; Wurster et al., 2016), then the Hall effect will increase the angular momentum in the disc if the magnetic field vector and rotation axis are initially antialigned, and will decrease the angular momentum if the two vectors are aligned (e.g., Krasnopolsky et al., 2011; Li et al., 2011; Braiding and Wardle, 2012a,b; Tsukamoto et al., 2015a, 2017; Wurster et al., 2016, 2018a,c).

\subsection{Calculating the Non-ideal MHD Coefficients}

When using the modified induction equation as given in Equation 5, the dependencies of the non-ideal coefficients are $\eta \equiv$ $\eta\left(\rho_{\text {gas }}, T_{\text {gas }}, B, n_{j}, m_{j}, e Z_{j}\right)$ for an arbitrary number of species, where $n_{j}, m_{j}$ and $e Z_{j}$ are the number density, mass and electric charge of species $j$, and only the charged species (i.e., $e Z_{j} \neq 0$ ) contribute to $\eta$; the equations can be found in Wardle and $\mathrm{Ng}$ (1999), Wardle (2007) and other papers, and thus will not be repeated here.

The species to be included in the calculation must be selected in advance, with the reaction rates between them determined experimentally or estimated theoretically (e.g., as presented in the UMIST Database; McElroy et al., 2013). The ionization sources must also be selected in advance, with the primary source for disc formation in typical molecular clouds being cosmic rays. The cosmic ray ionization rate is given by $\zeta_{\mathrm{cr}} \approx \zeta_{\mathrm{cr}, 0} \exp \left(-\Sigma / \Sigma_{\mathrm{cr}}\right)$, where $\Sigma_{\mathrm{cr}}$ is the characteristic column density for the attenuation of cosmic rays and $\zeta_{\mathrm{cr}, 0}$ is the unattenuated cosmic ray ionization rate. The latter has a canonical value of $\zeta_{\mathrm{cr}, 0}=10^{-17} \mathrm{~s}^{-1}$ for the Milky Way ISM (Spitzer and Tomasko, 1968; Umebayashi and Nakano, 1981). The cosmic ray ionization rate can vary from one region to another in the Galaxy (e.g., it is expected to be higher near a supernova remnant that can accelerate cosmic rays) and be modified by propagation effects, such as magnetic mirroring (e.g., Chandran, 2000; Padovani et al., 2009). As a cloud core collapses and a protostellar disc forms, the inner regions of the disc are shielded from cosmic rays (Padovani et al., 2014), thus other ionization sources become important, such as ionization by X-rays and energetic particles from young stellar objects, and ionization by radionuclide decay. Canonical X-rays are slightly less energetic and have a shorter attenuation depths than cosmic rays, thus will only affect the surface of the disc (e.g., Igea and Glassgold, 1999; Turner and Sano, 2008). The ionization from radionuclide decay, however, has rates ranging from $\zeta_{\mathrm{r}} \approx 10^{-23}$ to $10^{-18} \mathrm{~s}^{-1}$ depending on the ionization source (e.g., Kunz and Mouschovias, 2009; Umebayashi and Nakano, 2009) and can persist throughout the disc. Thus, ionization from radionuclide decay may be the dominant ionization source near the midplane of the disc.

Along with ions, molecular clouds include dust grains, which are important in coupling the magnetic field to the gas (Nishi et al., 1991). These are typically included in the numerical calculations of $\eta$ assuming a single grain population with a fixed radius, fixed bulk density, and a fixed gas-to-dust ratio. However, the grain size greatly affects the strength of the non-ideal effects; for example, Wurster et al. (2018b) showed that smaller grains tend to yield larger non-ideal MHD coefficients (see their Figure 2). However, molecular clouds do not contain a single grain size; one commonly used size distribution is the MRN (Mathis et al., 1977) grain distribution, $\mathrm{d} n_{\mathrm{g}}(a) / \mathrm{d} a \propto n_{\mathrm{H}} a^{-3.5}$, where $n_{\mathrm{H}}$ is the number density of the hydrogen nucleus and $n_{\mathrm{g}}(a)$ is the number density of grains with a radius smaller than $a$ (Draine and Lee, 
1984). The non-ideal MHD coefficients are sensitive to the upper and lower limits of the distribution. In particular, removing the very small grains of $\sim 10$ to a few $100 \AA$ from the distribution would increase the ambipolar diffusivity by $\sim 1-2$ orders of magnitude at number densities below $10^{10} \mathrm{~cm}^{-3}$ (Zhao et al., 2016).

As the non-ideal coefficients increase in value (either by increasing local density, magnetic field strength, or decreasing ionization rate), the numerical timestep required for numerical stability decreases (e.g., Mac Low et al., 1995; Choi et al., 2009; Bai, 2014; Wurster et al., 2014, 2018b). The two-dimensional simulations of Kunz and Mouschovias (2009) and Dapp et al. (2012) include all the above discussed ionization mechanisms to allow for a very low ionization fraction, and end their calculations prior to the timestep becoming prohibitively small. However, this is not currently computationally possible for global threedimensional simulations of disc formation and evolution. Thus, as a crude approximation, studies typically use $\zeta=\zeta_{\mathrm{cr}, 0}=10^{-17}$ $\mathrm{s}^{-1}$.

There are several private algorithms (e.g., Nakano et al., 2002; Kunz and Mouschovias, 2009; Okuzumi, 2009; Dapp et al., 2012; Tsukamoto et al., 2015b; Zhao et al., 2016, 2018; Higuchi et al., 2018) and publicly available codes (e.g Marchand et al., 2016; Wurster, 2016) that solve chemical networks of varying complexity to calculate the number density of each species, which can then be used to self-consistently calculate the nonideal MHD coefficients. These algorithms include ionization and reconnection amongst the included species (including dust), and ionization from cosmic rays. The results are expectedly dependent on the complexity of the networks and the input parameters, however, there is broad qualitative agreement among them. However, a direct comparison is difficult due to the parameter dependence, and even a single algorithm can produce widely varying results with small changes to its input parameters (e.g., the assumed dust grain properties).

\subsection{Numerical Models}

Ohmic resistivity was the first non-ideal effect to be included in analytical and numerical studies in attempts to prevent the magnetic braking catastrophe. This was followed by ambipolar diffusion, and finally the Hall effect. As discussed below, various studies reached various conclusions. However, recent three-dimensional radiation magnetohydrodynamical simulations (Tsukamoto et al., 2015a, 2017; Wurster et al., 2016, 2018c) have suggested that the Hall effect can prevent the magnetic braking catastrophe.

\subsubsection{Ohmic Resistivity and Ambipolar Diffusion}

A first attempt to solve the magnetic braking catastrophe was by Shu et al. (2006). They derived the equations governing the gravitational collapse of a molecular cloud core in the presence of a magnetic field, and included the effects of Ohmic diffusion. They noted that in order to reduce the magnetic field strength near the central stellar object to a value consistent with meteoritic evidence, $\eta_{\mathrm{O}} \approx 2 \times 10^{20} \mathrm{~cm}^{2} \mathrm{~s}^{-1}$ is required, which is a few orders of magnitude larger than estimated from kinetic theory (Shu et al., 2006). A similar calculation by Krasnopolsky et al. (2010) was able to reduce the required value using different assumptions, but $\eta_{\mathrm{O}}$ remained uncomfortably high. Recent calculations of $\eta_{\mathrm{O}}$ using chemical networks of varying complexity have shown that this value can be reached in the center of the first hydrostatic core during the first core phase (e.g., Marchand et al., 2016; Wurster, 2016). However, this high value persists over a very small $\rho-T$ phase-space, and values much lower than this are expected in the protostellar disc and background medium.

Contradicting the results of Shu et al. (2006) and Krasnopolsky et al. (2010), the three-dimensional numerical studies of Inutsuka et al. (2010) and Machida et al. (2011) formed protostellar discs; the latter formed a disc of $r \gtrsim 100$ au and their simulations used sink particles and the barotropic equation of state and were evolved until most of the envelope (i.e., the gas that was initially in the cloud core) had been accreted. As the envelope was accreted, the Alfvén waves became less efficient at transporting angular momentum from the gas near the protostar to the remaining envelope (simply because there was not much gas remaining in the envelope, as pointed out earlier in Mellon and $\mathrm{Li}, 2008)$. With less envelope and hence less magnetic braking, a $\sim 40$ au protostellar disc formed by $\sim 10^{4}$ yr which grew to $\sim 100$ au by $\sim 10^{5}$ yr. A similar study by Wurster et al. (2016) found $\sim 10$ au discs formed after $\sim 3 \times 10^{4} \mathrm{yr}$; this smaller disc was likely a result of a lower Ohmic resistivity and a larger sink particle. Even smaller Ohmic-enabled discs were found in the semi-analytic calculations of Dapp and Basu (2010) and Dapp et al. (2012).

Ohmic resistivity is expected to become important at the highest densities, especially late in the star formation process. However, by this stage, unless $\eta_{\mathrm{O}}$ was much higher than expected early on or the envelope was mostly depleted, the magnetic field could have already extracted enough angular momentum from the less dense regions to prevent discs from forming.

This possibility provides a strong motivation to study ambipolar diffusion, which was expected to be important in the early stages of star formation when the density of the molecular cloud core was still relatively low. This had long been included in cloud core formation models as a method to diffuse the magnetic field to initiate the quasi-static formation in an initially magnetically subcritical cloud and the later collapse of the cloud core (as first demonstrated by Mouschovias, 1976, 1977, 1979). On the smaller scales regarding protostar formation, it was hoped that this process could diffuse out enough of the magnetic field to permit a Keplerian disc to form. Analytical calculations by Hennebelle et al. (2016) predicted that an $\sim 18$ au disc should form when accounting for ambipolar diffusion for parameters typical of molecular cloud cores (see their Equation 13); this is much smaller than the 100 au-scale discs that would typically form in the absence of a magnetic field (see also Equation 14 of Hennebelle et al., 2016).

Numerical simulations have not provided a consensus regarding the effect of ambipolar diffusion. Mellon and $\mathrm{Li}$ (2009) re-performed their ideal MHD study from Mellon and Li (2008) but included ambipolar diffusion. Using two-dimensional axisymmetric models, they concluded that ambipolar diffusion alone did not weaken the magnetic braking enough to allow the formation of a disc that is resolvable by their simulations. Their conclusion held even when using a cosmic ray ionization rate ten times lower than canonical (i.e., making the system 
more neutral). However, their spherical-polar numerical domain included a 6.7 au hole at the center, thus discs below this radius could necessarily not form, and the artificial boundaries may have suppressed disc formation at radii even slightly larger than the boundary (for comment on effect of sink sizes, see Machida et al., 2014).

In the three-dimensional studies of Masson et al. (2016), Keplerian discs formed during the first hydrostatic core phase in their models that included ambipolar diffusion. Using a mass-toflux ratio of 5 times critical and an initial rotation of $\beta_{\text {rot }}=0.02$ (the ratio of rotational energy to gravitational potential energy), a disc of $\sim 80$ au formed; for similar initial conditions, small, disclike structures formed at late times when using ideal MHD. By inclining the initial magnetic field (c.f. section 3.2) by $\theta=40^{\circ}$ in the ideal MHD models, there was negligible effect on disc formation, leading them to conclude that ambipolar diffusion was more important than inclination in terms of disc formation. In their models with ambipolar diffusion, the discs formed more easily and were more massive in the non-aligned case.

Figure 6 shows the angular momentum in the first hydrostatic core (i.e., the gas that would collapse to form a rotationally supported disc if enough angular momentum is present) for three three-dimensional models by Tsukamoto et al. (2015b). After the first core has formed, there is least angular momentum in the ideal MHD model; there is approximately twice as much angular momentum when Ohmic resistivity is included, and approximately 5 times more when both Ohmic resistivity and ambipolar diffusion are included. In their model with Ohmic resistivity + ambipolar diffusion, a small $\sim 1$ au disc formed. At a similar time in the model with Ohmic resistivity + ambipolar diffusion by Tomida et al. (2015), a centrifugally supported disc of radius $\sim 5$ au formed, although it did not have a Keplerian profile; in their counterpart model that only included Ohmic resistivity, the centrifugally supported disc was $\sim 1$ au.

Although the disc in Tomida et al. (2015) is only $\sim 5$ au and remains approximately constant in size throughout their simulation, they expect it to grow with time as the envelope is depleted, as discussed above and in Tomida et al. (2013). By the end of the simulation (approximately 1 year after the formation of the stellar core), the disc is supported by the centrifugal force with substantial contribution from the gas pressure. At this time, the disc is rotating rapidly enough that it has triggered the gravito-rotational instability (e.g., Toomre, 1964; Bate, 1998; Saigo and Tomisaka, 2006; Saigo et al., 2008) and become nonaxisymmetric.

In a followup study, Tomida et al. (2017) modeled the long term evolution using sink particles. In agreement with their previous work, the disc stays small at early times due to efficient magnetic braking. As the disc evolves, magnetic braking becomes less efficient both due to the magnetic field dissipating in the disc and the dissipation of the envelope. Eventually, the disc becomes unstable, forming an $m=2$ perturbation (see also Hennebelle et al., 2016). The gravitational torques become more efficient at transporting angular momentum than the magnetic fields, thus control the future evolution of the disc. By the end of the Class I phase, the disc radius is in excess of 200 au.

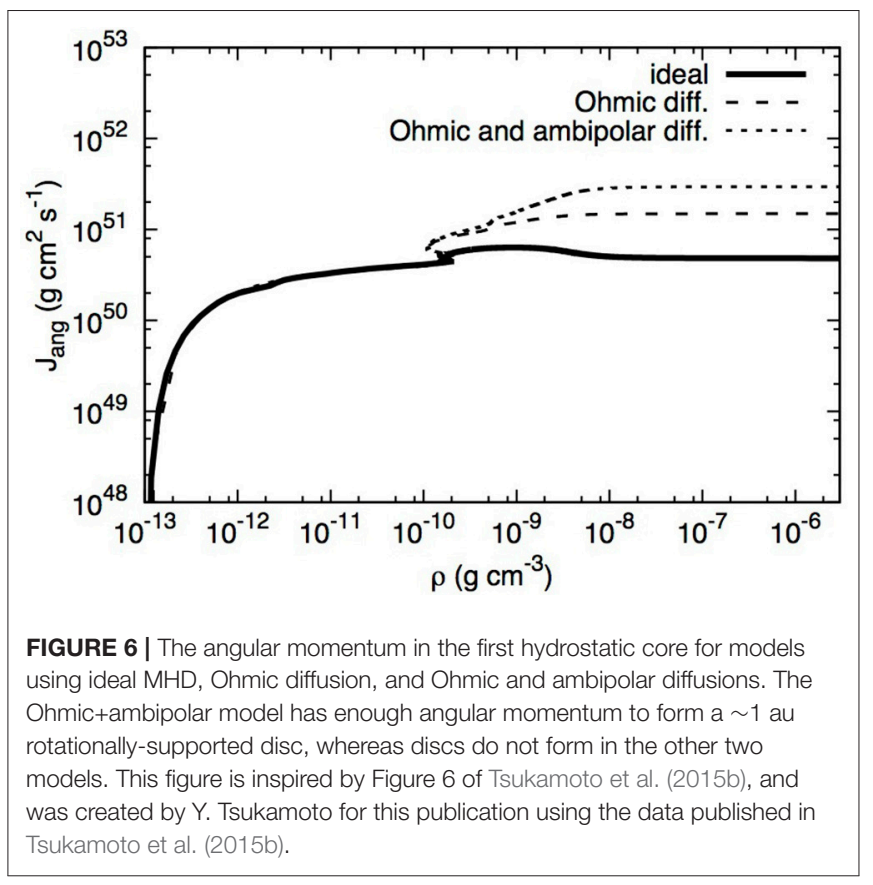

Vaytet et al. (2018) modeled the collapse to the stellar core including Ohmic resistivity + ambipolar diffusion. During the first core phase, the gas is funneled into the core along two dense filaments that arise from an initial $m=2$ perturbation. The disclike structure in their ideal MHD model is "puffier" than their resistive counterpart, but neither model appears to form a disc. One month after the formation of the stellar core, they find a "second core disc" of radius $r \lesssim 0.1$ au that has a Keplerian velocity profile.

Zhao et al. $(2016,2018)$ stressed the importance of the grain size on the ambipolar diffusivity (see also Dapp et al., 2012). They explored a range of grain sizes, initial magnetic field strengths and rotation rates, and found that rotationally supported structures often form early around the stellar seed but disappear at later times. Such structures can persist and grow to sizes of 20-40 au even for a rather strong initial magnetic field corresponding to a dimensionless mass-to-flux ratio of $\mu_{0}=2.4$ when the very small grains are removed from an MRN size distribution. Whether such grains are indeed removed or not, through, e.g., grain coagulation, remain to be determined.

\subsubsection{Complete Non-ideal MHD Description}

Including the Hall effect in numerical models becomes computationally expensive due to the small timestep constraint (e.g., Sano and Stone, 2002a; Bai, 2014; Tsukamoto et al., 2015a; Wurster et al., 2016, 2018b), thus, at the time of writing, there are only a few global two-dimensional (Krasnopolsky et al., 2011; Li et al., 2011) and three-dimensional (Tsukamoto et al., 2015a, 2017; Wurster et al., 2016, 2018a,c,d) global disc-formation studies that include this process.

The first simulation to include the Hall effect in a global simulation was Krasnopolsky et al. (2011). This simulation was a two-dimensional axisymmetric Eulerian simulation that 
included gravity of a pre-formed protostar but no self-gravity amongst the gas. The Hall coefficient, $\eta_{\mathrm{H}}$, was set as a constant positive value, which is the opposite sign as found by later studies (e.g., Tsukamoto et al., 2015a; Marchand et al., 2016; Wurster, 2016; Wurster et al., 2016). They formed rotationally supported discs in all models that included the Hall effect, except for their model with the weakest resistivity.

Figure 7 shows the rotational profiles for three models in the study by Krasnopolsky et al. (2011), where they varied the initial alignment of the rotation and magnetic field vectors. In the outer regions of their models, the Hall effect is too weak to have any significant effect on the evolution of the gas, thus the gas simply follows the initial velocity profile combined with the effect of gravity. In the inner region of all three models, the Hall effect spins up the gas to reach a Keplerian profile. In the aligned model (left panel), the Hall effect contributes to the initial rotation, whereas in the anti-aligned case (right panel), it detracts from it; in the anti-aligned case, the Hall velocity is strong enough to cancel out the initial rotational profile and form a counter-rotating disc with a Keplerian profile.

In their followup study, Li et al. (2011) self-consistently calculated the value for $\eta_{\mathrm{H}}$, which now necessarily varied in space and time; $\eta_{\mathrm{H}}<0$, and the maximum of its absolute value was lower than the value used in their previous study. Given their parameter space, no model formed a rotationally supported disc. The Hall effect did spin up the material of the gas near the protostar, but it was not significant enough to reach Keplerian velocities.

Subsequent studies led by Tsukamoto and Wurster modeled the three-dimensional collapse of a molecular cloud core through to at least the end of the first core phase. The models typically included self-consistent calculations of the non-ideal MHD coefficients, included flux limited diffusion and excluded sink particles ${ }^{3}$. When using the canonical, unattenuated cosmic ray ionization rate of $\zeta_{\mathrm{cr}}=10^{-17} \mathrm{~s}^{-1}$, Tsukamoto et al. (2015a) and Wurster et al. (2018c) find that rotationally supported discs form if the magnetic field and rotation vectors are anti-aligned, whereas no disc forms if they are aligned. The top row in Figure 8 shows the gas column density for ideal MHD, nonideal MHD and hydrodynamic models in the midplane during the first hydrostatic core phase. In both studies (Tsukamoto et al., 2015a; Wurster et al., 2018c), the disc has a radius of $r \sim 25 \mathrm{au}$, and becomes bar-unstable during the first core phase. For comparison, a purely hydrodynamics model formed a disc of $\sim 60 \mathrm{au}$, and this disc is formed even earlier during the first core phase due to the lack of magnetic support. Expectedly, the purely hydrodynamic disc has the largest angular momentum in the first core and/or disc (see Figure 9). However, the Hall effect in the anti-aligned non-ideal model decreases magnetic braking, permitting the angular momentum to remain in the gas near/in the first core, and hence permitting the disc to form. The angular momentum in the disc is approximately half that of the purely hydrodynamics model (Wurster et al., 2018c). By aligning the magnetic field and the rotation axis, the angular momentum

\footnotetext{
${ }^{3}$ Wurster et al. (2016) used 6.7 au sink particles together with a barotropic equation of state in their disc formation study using PHANTOM (Price et al., 2018).
}

again decreases by a factor of $\sim 12$, to a value too low for a disc to form.

The models presented in Figures 6, 9 use slightly different initial conditions, however, both ideal MHD models have similar final angular momenta in their first cores, thus can be reasonably compared. By including Ohmic resistivity and ambipolar diffusion, the angular momentum in the first core increases to $L_{\mathrm{fc}} \sim 2.5 \times 10^{51} \mathrm{~g} \mathrm{~cm}^{2} \mathrm{~s}^{-1}$ (dotted line in Figure 6), which is higher than when the three non-ideal terms are added in the aligned orientation (double-dot line in Figure 9). Therefore, some of the angular momentum gain caused by Ohmic resistivity and ambipolar diffusion is lost by including the Hall effect in an aligned orientation. As previously discussed, additional angular momentum is gained by the Hall effect in the anti-aligned case. Summarily, the order of angular momenta in the first core is $L_{\mathrm{fc}}($ ideal MHD $)<L_{\mathrm{fc}}(\mathrm{Ohmic}+$ ambipolar+Hall; aligned $)<$ $L_{\mathrm{fc}}(\mathrm{Ohmic}+$ ambipolar $)<L_{\mathrm{fc}}(\mathrm{Ohmic}+$ ambipolar+Hall; antialigned $)<L_{\mathrm{fc}}$ (Hydrodynamics).

Given that the anti-aligned non-ideal model formed a large disc while the aligned model did not, Tsukamoto et al. (2015a) proposed that there should be a bi-modality in the population of discs around stars-that is, approximately half of the Class 0 objects should have protostellar discs. However, this assumption was made assuming the magnetic field was either aligned or anti-aligned with the rotation axis. Since there is observational evidence that the magnetic field appears to be randomly orientated with respect to the rotation axis, at least on the 1000 au scale (Hull et al., 2013), Tsukamoto et al. (2017) modeled various angles between the rotation axis and the magnetic field. The angular momentum in the first core is shown in Figure 10 for various initial orientations, and it differs by an order of magnitude between the two extreme angles: $\theta=0$ and $180^{\circ}$. Changing the angle by $45^{\circ}$ from either extreme value has a minimal effect on the angular momentum evolution, and even a change of $70^{\circ}$ only changes the final angular momentum by a factor of a few. Thus, the models with $\theta \neq 90^{\circ}$ have angular momenta evolutions that are similar to either $\theta=0$ or $180^{\circ}$, suggesting that, even if the initial magnetic fields are randomly orientated with the rotation axis, the bi-modality in disc sizes should exist, with large discs forming for $\theta \gtrsim 100^{\circ}$ and negligible discs forming for $\theta \lesssim 80^{\circ}$.

The magnetic field strength and plasma $\beta$ for an ideal model and two non-ideal MHD models are shown in bottom two rows of Figure 8. As expected, the magnetic field is dragged into the core of the ideal MHD model, thus its first hydrostatic core has a very strong field strength. However, in the non-ideal cases, the neutrals can slip through the magnetic field, thus a dense core forms, but its central magnetic field strength is weaker than in the ideal MHD model. Unlike the neutrals, the velocities of the charged particles decrease and they approach the core due to the non-ideal effects. This allows the charged particles, and the magnetic flux they drag in, to pileup in a torus around the core. This torus of charged particles expands outwards in radius as more charged particles pile up, further enhancing the magnetic field. This is a demonstration of a "magnetic wall," which is thoroughly discussed in the two-dimensional models of Tassis and Mouschovias (2005b) and first predicted to exist analytically 

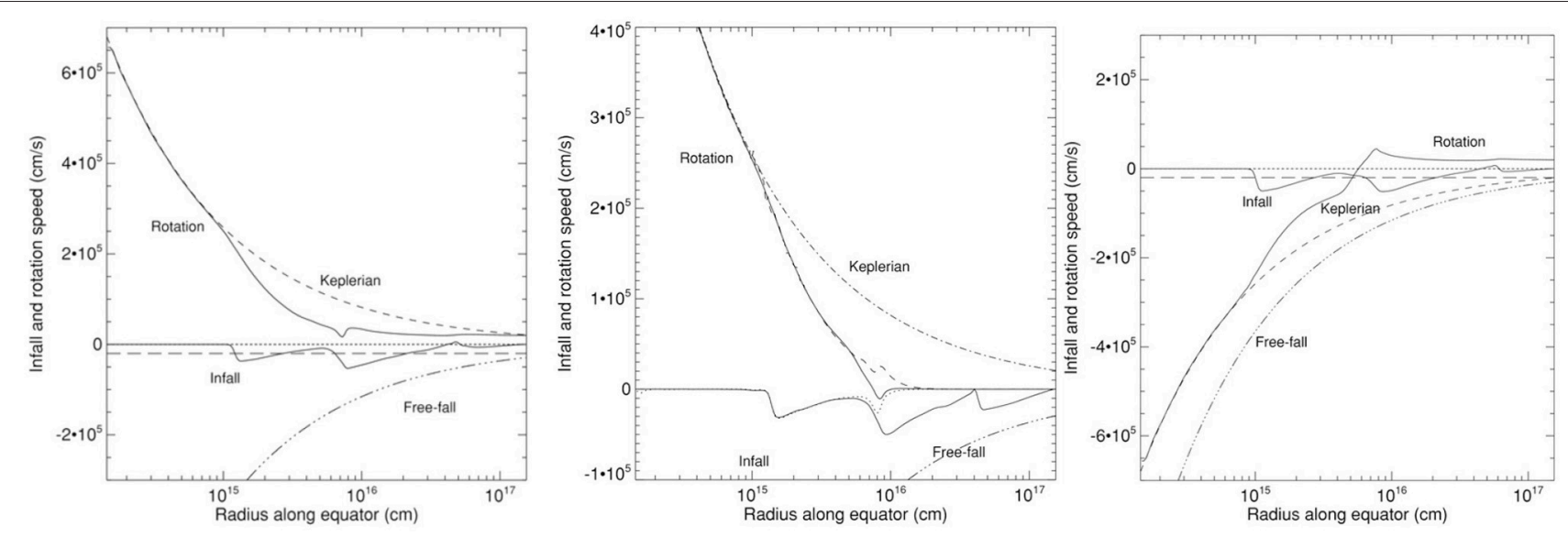

FIGURE 7 | Rotational profiles for aligned, no rotation and anti-aligned two-dimensional models. The initial rotation in the rotating models is $v_{\phi}=-2 \times 10^{4} \mathrm{~cm} \mathrm{~s} \mathrm{~s}^{-1}$. The gas in the outer regions is governed by the initial conditions, while Keplerian discs of $r \sim 10^{15} \mathrm{~cm}$ form in all three models. These are Figures $1 \mathrm{~b}, 3 \mathrm{~b}$, and $2 \mathrm{~b}$ of Krasnopolsky et al. (2011). ${ }^{\oplus}$ AAS. Reproduced with permission.

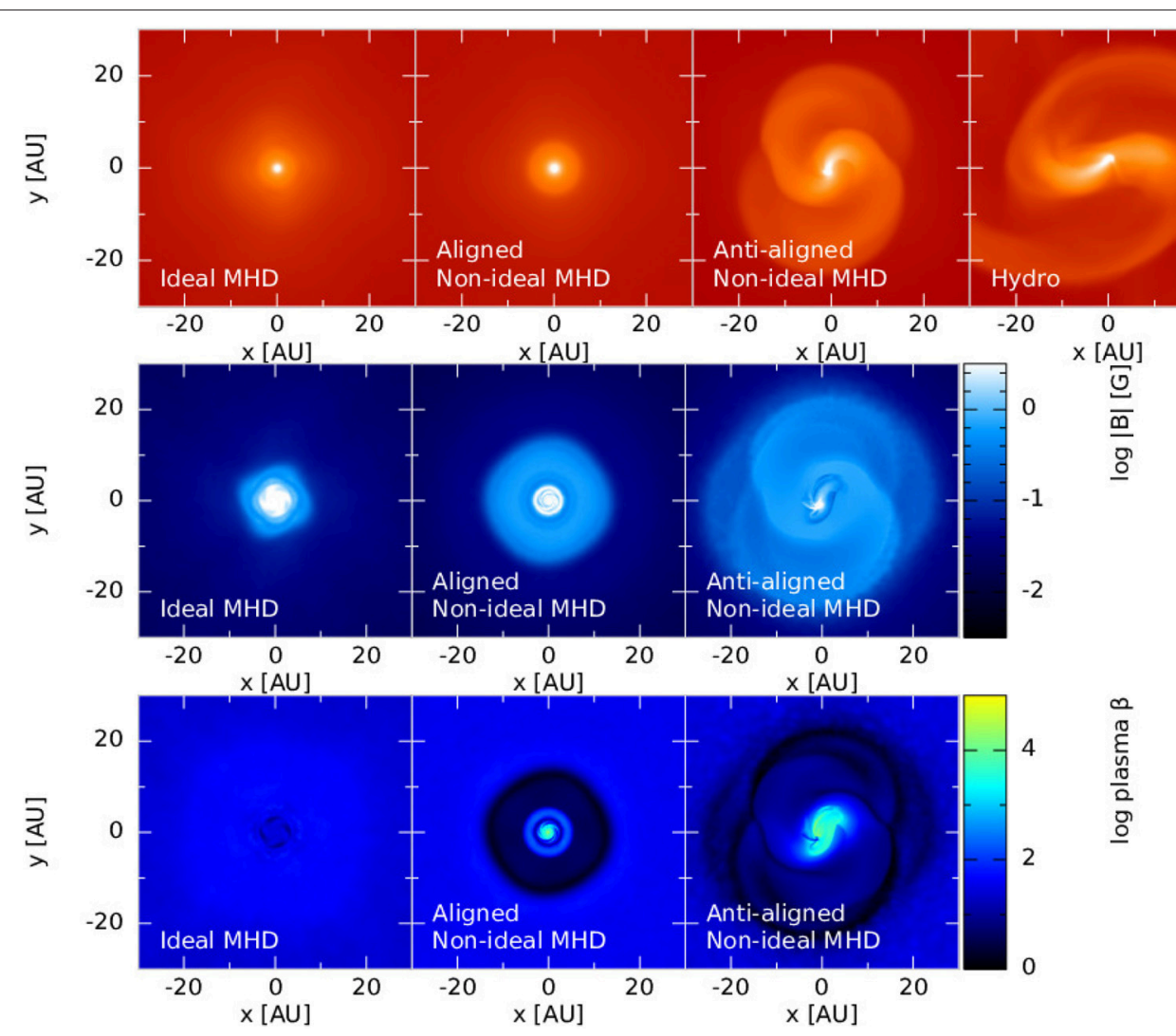

FIGURE 8 | The gas column density (top), magnetic field strength (middle) and plasma $\beta$ (bottom) in a slice through the mid-plane of an ideal MHD model, two non-ideal MHD models (Ohmic+ambipolar+Hall; $\zeta \mathrm{cr}=10^{-17} \mathrm{~s}^{-1}$ ) with different initial orientations of the magnetic field, and purely hydrodynamics model (top row only). The snapshots are near the end of the first core phase. When the rotation and magnetic field vector are aligned, the non-ideal MHD model is similar to the ideal MHD model with no disc, but when the vectors are anti-aligned, a gravitationally unstable disc forms, similar to the hydrodynamics model. The maximum magnetic field strength is in the disc for the anti-aligned non-ideal model, while it is in the center of the first core for the remaining two models. The disc in the anti-aligned model is supported by gas pressure. The figure is adapted/inspired by Figures 1 and 8 of Wurster et al. (2018c) and Figure 1 of Tsukamoto et al. (2015a) and was created for this publication using the data from Wurster et al. (2018c). 


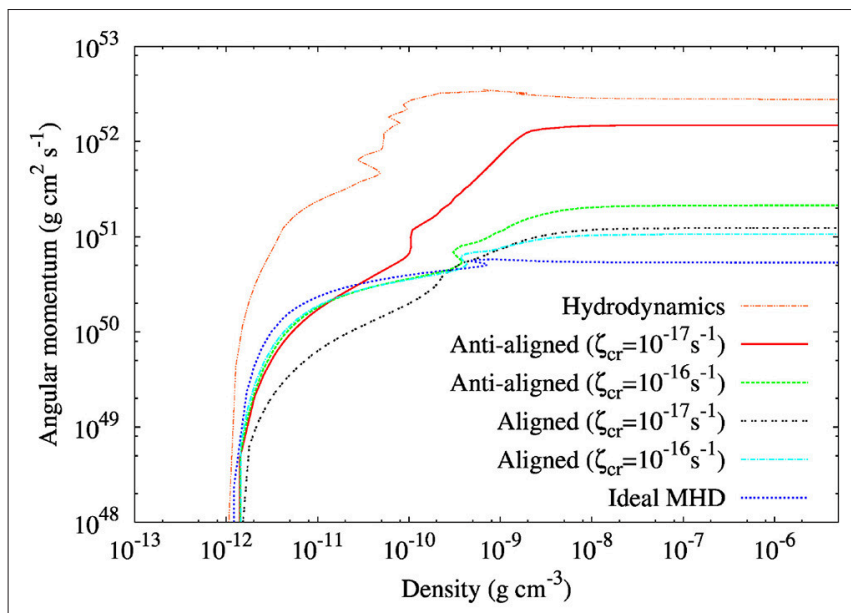

FIGURE 9 | The angular momentum in the first hydrostatic core which collapses to form a disc for models with various cosmic ray ionization rates, $\zeta \mathrm{cr}$. Only the purely hydrodynamics and the anti-aligned $\left(\zeta_{\mathrm{cr}}=10^{-17} \mathrm{~s}^{-1}\right)$ models form discs, both of which become bar-unstable. The angular momentum of the ideal MHD model differs slightly from that shown in Figure 6 due to different initial conditions. This is adapted from Figure 2 of Wurster et al. (2018c), and was created for this publication using the data from Wurster et al. (2018c).

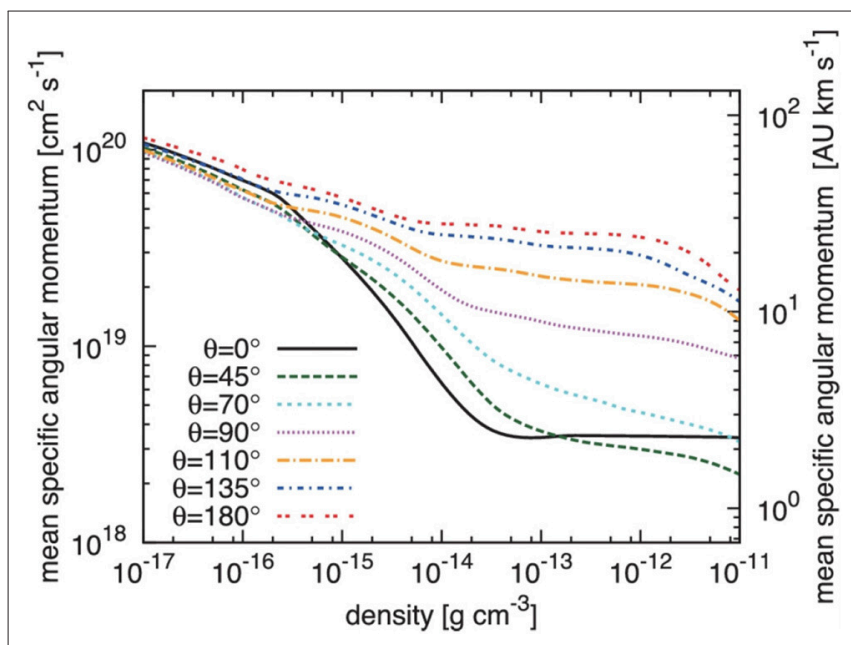

FIGURE 10 | The angular momentum in the first hydrostatic core which collapses to form a disc for non-ideal MHD models at various angles $\theta$ between the rotation axis and magnetic field vector; $\theta=0^{\circ}$ represents the aligned case. Except for the $\theta=90^{\circ}$ case, the angular momenta are grouped around $\theta=0^{\circ}$ and $\theta=180^{\circ}$, suggesting a bi-modality in disc sizes. This figure is Figure 6 of Tsukamoto et al. (2017), and is reproduced with permission.

by Li and McKee (1996). In the non-ideal MHD models shown above, the magnetic wall occurs at $r \sim 1-3$ au from the center of the core.

In the domain presented in Figure 8, plasma $\beta>1$, indicating that gas pressure is greater than magnetic pressure everywhere. The disc in the anti-aligned model has plasma $\beta>100$ due to the rotationally supported disc; in the disc, magnetic diffusion is efficient and the magnetic flux is removed from the region
(Tsukamoto et al., 2015a). In the center of the aligned model, plasma $\beta$ becomes high, but this region is small enough that disc formation is not possible. Finally, the strong magnetic field in the ideal simulations yields plasma $\beta<100$ over the entire computational domain, again iterating the importance of magnetic fields in that model. Similar results with slightly lower values of plasma $\beta$ are reached in Tsukamoto et al. (2015a, see their Figure 1), since their initial magnetic field strength is stronger than in the figures presented here.

These two studies discussed here, Tsukamoto et al. (2015a) and Wurster et al. (2018c), both modeled the gravitational collapse of a $1 \mathrm{M}_{\odot}$ molecular cloud core, however many of the remaining initial parameters were different, including the initial rotation rate, radius, magnetic field strength, and microphysical properties that controlled the coefficients of the non-ideal MHD terms. Thus, gravitationally unstable discs forming around the time of the first core in anti-aligned models that include the Hall effect is a robust result.

Concurrent to the study of Tsukamoto et al. (2015a), Wurster et al. (2016) studied the evolution of the disc until $\sim 5,000 \mathrm{yr}$ after the formation of the protostar; these simulations included sink particles and the barotropic equation of state. In their aligned models that include, respectively, only the Hall effect and all three non-ideal MHD terms, no discs formed. In their antialigned models, $\sim 38$ and $\sim 15$ au discs formed, respectively. The disc radius in the non-ideal model with the three terms remained approximately constant until the end of the simulation, while the model with only the Hall effect decreased slightly to $\sim 20$ au by 5,000 yr. Neither disc formed a bar-mode instability, since this instability would be stabilized by the sink particle. The disc in the non-ideal model is smaller than the $\sim 25$ au discs from the previous studies, but both sink particles and the barotropic equation of state have been shown to reduce the disc sizes compared to models that exclude sink particles and include radiation hydrodynamics (e.g., Tomida et al., 2013; Machida et al., 2014; Lewis and Bate, 2018; Wurster et al., 2018c).

Finally, the effect of the cosmic ray ionization rate was studied in Wurster et al. (2018a). When the ionization rate is increased by even a factor of ten compared to the canonical value for the interstellar medium, the angular momentum in the first core is low enough such that no discs form, independent of magnetic field orientation. This indicates the importance of the value of the cosmic ray ionization rate: if it is too high, then rotationally supported discs cannot form, despite the initial conditions. This may have implications for, e.g., starburst galaxies (e.g., Bisbas et al., 2015) or the Galactic Center (e.g., Oka et al., 2005) where the cosmic ray ionization rate is $10-100$ times higher than the canonical value of $\zeta_{\mathrm{cr}, 0}=10^{-17} \mathrm{~s}^{-1}$.

\section{CONCLUSION}

Protostellar discs have been observed around young stars at all stages of evolution, and naturally form alongside their host star. The star forming environment has strong magnetic fields, low ionization fractions, and non-laminar velocity flows. The focus of this article is on how the magnetic field affects the formation 
of disc in lightly ionized molecular cloud cores where non-ideal MHD effects are important.

Early numerical simulations of star formation that include strong magnetic fields under the ideal MHD approximation fail to form discs during the star forming process; this is known as the magnetic braking catastrophe. Given that discs are observed, this highlighted the need for including additional physical ingredients in the simulations. Several studies continued using the ideal MHD approximation, but made their initial conditions less idealized. By misaligning the magnetic field and rotation vectors, larger or smaller discs could form depending on the study, hence likely depending on the initial conditions. The misalignment can be naturally induced by a turbulent velocity field, which can speed up the removal of magnetic flux near the center and thus promote disc formation through enhanced magnetic reconnection; however, increasing turbulence has also been numerically observed to hinder disc formation. Given that reconnection in ideal MHD simulations is necessarily realized numerically, this process needs to be evaluated more carefully.

Molecular clouds are observed to be mostly neutral, and the interaction between neutral and charged particles gives rise to three non-ideal effects: Ohmic resistivity, ambipolar diffusion and the Hall effect. When ambipolar diffusion is included in numerical simulations, small discs of $1-5$ au are expected to form over an extended period of time; even larger discs can form if very small grains are removed from the grain size distribution. When the Hall effect is included and the magnetic field vector is antialigned with the rotation axis, larger discs of 25 au or more form. However, these results may be subject to initial conditions and the choice of parameters such as dust grain properties or ionization rates.

In summary, considerable progress has been made in averting the magnetic braking catastrophe, through turbulence and

\section{REFERENCES}

Allen, A., Li, Z.-Y., and Shu, F. H. (2003). Collapse of magnetized singular isothermal toroids. II. Rotation and magnetic braking. Astrophys. J. 599, 363379. doi: $10.1086 / 379243$

Alves, F. O., Girart, J. M., Padovani, M., Galli, D., Franco, G. A. P., Caselli, P., et al. (2018). Magnetic field in a young circumbinary disk. Astron. Astrophys. 616:A56. doi: 10.1051/0004-6361/201832935

Andersson, B.-G., Lazarian, A., and Vaillancourt, J. E. (2015). Interstellar dust grain alignment. Annu. Rev. Astron. Astrophys. 53, 501-539. doi: 10.1146/annurev-astro-082214-122414

Babcock, H. W., and Cowling, T. G. (1953). General magnetic fields in the Sun and stars (Report on progress of astronomy). Month. Notices of the RAS 113, 357-381. doi: 10.1093/mnras/113.3.357

Bacciotti, F., Girart, J. M., Padovani, M., Podio, L., Paladino, R., Testi, L., et al. (2018). ALMA observations of polarized emission toward the CW Tau and DG Tau protoplanetary disks: constraints on dust grain growth and settling. Astrophys. J. 865:L12. doi: 10.3847/2041-8213/aadf87

Bai, X.-N. (2014). Hall-effect-controlled gas dynamics in protoplanetary disks. I. Wind solutions at the inner disk. Astrophys. J. 791:137. doi: 10.1088/0004-637X/791/2/137

Basu, S., and Mouschovias, T. C. (1994). Magnetic braking, ambipolar diffusion, and the formation of cloud cores and protostars. 1: axisymmetric solutions. Astrophys. J. 432, 720-741. doi: 10.1086/ 174611 related field-rotation misalignment in the ideal MHD limit, enhanced ambipolar diffusion, and especially the Hall effect in the case of anti-aligned magnetic field and rotation axis. Further progress is expected when these and other effects are taken into account together, especially in simulations that can run to the end of the protostellar accretion phase of star formation. These more comprehensive models will be guided by, and be used to interpret, the increasingly more detailed multi-scale observations of the gas kinematics and magnetic fields in the ALMA era.

\section{AUTHOR CONTRIBUTIONS}

JW and Z-YL were both responsible for writing and editing the manuscript.

\section{ACKNOWLEDGMENTS}

We would like to thank the referees for very thorough and insightful reports which have greatly contributed to the quality of this manuscript. JW acknowledges support from the European Research Council under the European Community's Seventh Framework Programme (FP7/2007- 2013 grant agreement no. 339248). Z-YL is supported in part by NASA NNX14AB38G, NASA 80NSSC18K1095 and NSF AST-1815784 and AST1716259. The calculations for this review were performed on the DiRAC Complexity machine, jointly funded by STFC and the Large Facilities Capital Fund of BIS (STFC grants ST/K000373/1, ST/K0003259/1 and ST/M006948/1), and the University of Exeter Supercomputer, a DiRAC Facility jointly funded by STFC, the Large Facilities Capital Fund of BIS, and the University of Exeter, and the RIVANNA computer cluster at the University of Virginia.

Basu, S., and Mouschovias, T. C. (1995). Magnetic braking, ambipolar diffusion, and the formation of cloud cores and protostars. III. Effect of the initial mass-to-flux ratio. Astrophys. J. 453:271. doi: 10.1086/176387

Bate, M. R. (1998). Collapse of a molecular cloud core to stellar densities: the first three-Dimensional calculations. Astrophys. J. 508, L95-L98. doi: $10.1086 / 311719$

Bate, M. R. (2010). Collapse of a molecular cloud core to stellar densities: the radiative impact of stellar core formation on the circumstellar disc. Month. Notices RAS 404, L79-L83. doi: 10.1111/j.1745-3933.2010.00839.x

Bate, M. R. (2011). Collapse of a molecular cloud core to stellar densities: the formation and evolution of pre-stellar discs. Month. Notices RAS 417, 20362056. doi: 10.1111/j.1365-2966.2011.19386.x

Bate, M. R., Tricco, T. S., and Price, D. J. (2014). Collapse of a molecular cloud core to stellar densities: stellar-core and outflow formation in radiation magnetohydrodynamic simulations. Month. Notices RAS 437, 77-95. doi: $10.1093 / \mathrm{mnras} / \mathrm{stt} 1865$

Bergin, E. A., and Tafalla, M. (2007). Cold dark clouds: the initial conditions for star formation. Annu. Rev. Astron. Astrophys. 45, 339-396. doi: 10.1146/annurev.astro.45.071206.100404

Bisbas, T. G., Papadopoulos, P. P., and Viti, S. (2015). Effective destruction of CO by Cosmic rays: implications for tracing $\mathrm{H}_{2}$ gas in the universe. Astrophys. J. 803:37. doi: 10.1088/0004-637X/803/1/37

Boss, A. P. (1993). Collapse and fragmentation of molecular cloud cores. I - Moderately centrally condensed cores. Astrophys. J. 410, 157-167. doi: $10.1086 / 172734$ 
Boss, A. P. (1997). Giant planet formation by gravitational instability. Science 276, 1836-1839. doi: 10.1126/science.276.5320.1836

Boss, A. P. (1999). Collapse and fragmentation of molecular cloud cores. VI. Slowly rotating magnetic clouds. Astrophys. J. 520, 744-750. doi: 10.1086/ 307479

Boss, A. P. (2002). Collapse and fragmentation of molecular cloud cores. VII. Magnetic fields and multiple protostar formation. Astrophys. J. 568, 743-753. doi: $10.1086 / 339040$

Boss, A. P. (2005). Collapse and fragmentation of molecular cloud cores. VIII. Magnetically supported infinite sheets. Astrophys. J. 622, 393-403. doi: $10.1086 / 428113$

Boss, A. P. (2007). Collapse and fragmentation of molecular cloud cores. IX. Magnetic braking of initially filamentary clouds. Astrophys. J. 658, 1136-1143. doi: $10.1086 / 512061$

Boss, A. P. (2009). Collapse and fragmentation of molecular cloud cores. X. Magnetic braking of prolate and oblate cores. Astrophys. J. 697, 1940-1945. doi: $10.1088 / 0004-637 \mathrm{X} / 697 / 2 / 1940$

Boss, A. P., and Myhill, E. A. (1995). Collapse and fragmentation of molecular cloud cores. III. Initial differential rotation. Astrophys. J. 451:218. doi: $10.1086 / 176213$

Bourke, T. L., Myers, P. C., Robinson, G., and Hyland, A. R. (2001). New $\mathrm{OH}$ zeeman measurements of magnetic field strengths in molecular clouds. Astrophys. J. 554, 916-932. doi: 10.1086/321405

Braiding, C. R., and Wardle, M. (2012a). The hall effect in star formation. Month. Notices RAS 422, 261-281. doi: 10.1111/j.1365-2966.2012.20601.x

Braiding, C. R., and Wardle, M. (2012b). The Hall effect in accretion flows. Month. Notices RAS 427, 3188-3195. doi: 10.1111/j.1365-2966.2012.22001.x

Chandran, B. D. G. (2000). Confinement and isotropization of galactic cosmic rays by molecular-cloud magnetic mirrors when turbulent scattering is weak. Astrophys. J. 529, 513-535. doi: 10.1086/308232

Choi, E., Kim, J., and Wiita, P. J. (2009). An explicit scheme for incorporating ambipolar diffusion in a magnetohydrodynamics code. Astrophys. J. 181, 413420. doi: 10.1088/0067-0049/181/2/413

Ciolek, G. E., and Mouschovias, T. C. (1993). Ambipolar diffusion, interstellar dust, and the formation of cloud cores and protostars. I. Basic physics and formulation of the problem. Astrophys. J. 418:774. doi: 10.1086/ 173435

Ciolek, G. E., and Mouschovias, T. C. (1994). Ambipolar diffusion, interstellar dust, and the formation of cloud cores and protostars. 3: typical axisymmetric solutions. Astrophys. J. 425, 142-160. doi: 10.1086/173971

Codella, C., Cabrit, S., Gueth, F., Podio, L., Leurini, S., Bachiller, R., et al. (2014). The ALMA view of the protostellar system HH212. The wind, the cavity, and the disk. Astron. Astrophys. 568:L5. doi: 10.1051/0004-6361/2014 24103

Commerçon, B., Hennebelle, P., Audit, E., Chabrier, G., and Teyssier, R. (2010). Protostellar collapse: radiative and magnetic feedbacks on small-scale fragmentation. Astron. Astrophys. 510:L3. doi: 10.1051/0004-6361/200913597

Cox, E. G., Harris, R. J., Looney, L. W., Li, Z.-Y., Yang, H., Tobin, J. J., et al. (2018). ALMA's polarized view of 10 protostars in the perseus molecular cloud. Astrophys. J. 855:92. doi: 10.3847/1538-4357/aaacd2

Cox, E. G., Harris, R. J., Looney, L. W., Segura-Cox, D. M., Tobin, J., Li, Z.-Y., et al. (2015). High-resolution $8 \mathrm{~mm}$ and $1 \mathrm{~cm}$ polarization of IRAS 4A from the VLA nascent disk and multiplicity (VANDAM) survey. Astrophys. J. 814:L28. doi: 10.1088/2041-8205/814/2/L28

Crutcher, R. M. (1999). Magnetic fields in molecular clouds: observations confront theory. Astrophys. J. 520, 706-713. doi: 10.1086/307483

Dapp, W. B., and Basu, S. (2010). Averting the magnetic braking catastrophe on small scales: disk formation due to Ohmic dissipation. Astron. Astrophys. 521:L56. doi: 10.1051/0004-6361/201015700

Dapp, W. B., Basu, S., and Kunz, M. W. (2012). Bridging the gap: disk formation in the Class 0 phase with ambipolar diffusion and Ohmic dissipation. Astron. Astrophys. 541:A35. doi: 10.1051/0004-6361/201117876

Davis, L. Jr., and Greenstein, J. L. (1951). The polarization of starlight by aligned dust grains. Astrophys. J. 114:206. doi: 10.1086/145464

Dent, W. R. F., Pinte, C., Cortes, P. C., Ménard, F., Hales, A., Fomalont, E., et al. (2019). Submillimetre dust polarisation and opacity in the HD163296 protoplanetary ring system. Month. Notices RAS. 482, L29-L33. doi: 10.1093/ mnrasl $/$ sly 181

Draine, B. T., and Lee, H. M. (1984). Optical properties of interstellar graphite and silicate grains. Astrophys. J. 285, 89-108. doi: 10.1086/162480
Duffin, D. F., and Pudritz, R. E. (2009). The early history of protostellar disks, outflows, and binary stars. Astrophys. J. 706, L46-L51. doi: 10.1088/0004-637X/706/1/L46

Fielding, D. B., McKee, C. F., Socrates, A., Cunningham, A. J., and Klein, R. I. (2015). The turbulent origin of spin-orbit misalignment in planetary systems. Month. Notices RAS 450, 3306-3318. doi: 10.1093/mnras/stv836

Galli, D., Lizano, S., Shu, F. H., and Allen, A. (2006). Gravitational collapse of magnetized clouds. I. Ideal magnetohydrodynamic accretion flow. Astrophys. J. 647, 374-381. doi: 10.1086/505257

Galli, D., and Shu, F. H. (1993). Collapse of magnetized molecular cloud cores. II. Numerical results. Astrophys. J. 417:243. doi: 10.1086/173306

Girart, J. M., Fernández-López, M., Li, Z.-Y., Yang, H., Estalella, R., Anglada, G., et al. (2018). Resolving the polarized dust emission of the disk around the massive star powering the HH 80-81 radio jet. Astrophys. J. 856:L27. doi: $10.3847 / 2041-8213 / a a b 76 b$

Goldsmith, P. F., and Arquilla, R. (1985). "Rotation in dark clouds," in Protostars and Planets II, eds D. C. Black and M. S. Matthews (University of Arizona Press), 137-149.

Goodman, A. A., Benson, P. J., Fuller, G. A., and Myers, P. C. (1993). Dense cores in dark clouds. VIII - Velocity gradients. Astrophys. J. 406, 528-547. doi: $10.1086 / 172465$

Gray, W. J., McKee, C. F., and Klein, R. I. (2018). Effect of angular momentum alignment and strong magnetic fields on the formation of protostellar discs. Month. Notices RAS 473, 2124-2143. doi: 10.1093/mnras/ stx2406

Hall, J. S. (1949). Observations of the polarized light from stars. Science 109, 166-167. doi: 10.1126/science.109.2825.166

Harris, R. J., Cox, E. G., Looney, L. W., Li, Z.-Y., Yang, H., Fernández-López, M., et al. (2018). ALMA observations of polarized $872 \mu \mathrm{m}$ dust emission from the protostellar systems VLA 1623 and L1527. Astrophys. J. 861:91. doi: $10.3847 / 1538-4357 /$ aac6ec

Heiles, C., and Crutcher, R. (2005). "Magnetic fields in diffuse HI and molecular clouds," in Cosmic Magnetic Fields, volume 664 of Lecture Notes in Physics, eds R. Wielebinski and R. Beck (Berlin: Springer Verlag), 137.

Hennebelle, P., and Ciardi, A. (2009). Disk formation during collapse of magnetized protostellar cores. Astron. Astrophys. 506, L29-L32. doi: 10.1051/0004-6361/200913008

Hennebelle, P., Commerçon, B., Chabrier, G., and Marchand, P. (2016). Magnetically self-regulated formation of early protoplanetary disks. Astrophys. J. 830:L8. doi: 10.3847/2041-8205/830/1/L8

Hennebelle, P., and Fromang, S. (2008). Magnetic processes in a collapsing dense core. I. Accretion and ejection. Astron. Astrophys. 477, 9-24. doi: 10.1051/0004-6361:20078309

Heyer, M. H., and Brunt, C. M. (2004). The universality of turbulence in galactic molecular clouds. Astrophys. J. 615, L45-L48. doi: 10.1086/425978

Higuchi, K., Machida, M. N., and Susa, H. (2018). Evolution of magnetic fields in collapsing star-forming clouds under different environments. Month. Notices RAS 475, 3331-3347. doi: 10.1093/mnras/sty046

Hiltner, W. A. (1949). On the presence of polarization in the continuous radiation of stars. II. Astrophys. J. 109:471. doi: 10.1086/145151

Hull, C. L. H., Plambeck, R. L., Bolatto, A. D., Bower, G. C., Carpenter, J. M., Crutcher, R. M., et al. (2013). Misalignment of magnetic fields and outflows in protostellar cores. Astrophys. J. 768:159. doi: 10.1088/0004-637X/768/2/159

Hull, C. L. H., Yang, H., Li, Z.-Y., Kataoka, A., Stephens, I. W., Andrews, S., et al. (2018). ALMA observations of polarization from dust scattering in the IM lup protoplanetary disk. Astrophys. J. 860:82. doi: 10.3847/1538-4357/aabfeb

Igea, J., and Glassgold, A. E. (1999). X-ray ionization of the disks of young stellar objects. Astrophys. J. 518, 848-858. doi: 10.1086/307302

Inoue, T., and Inutsuka, S.-i. (2008). Two-fluid magnetohydrodynamic simulations of converging $\mathrm{H}$ I flows in the interstellar medium. I. Methodology and basic results. Astrophys. J. 687, 303-310. doi: 10.1086/590528

Inoue, T., and Inutsuka, S.-i. (2009). Two-fluid magnetohydrodynamics simulations of converging H I flows in the interstellar medium. II. Are molecular clouds generated directly from a warm neutral medium? Astrophys. J. 704, 161-169. doi: $10.1088 / 0004-637 \mathrm{X} / 704 / 1 / 161$

Inoue, T., Inutsuka, S.-I., and Koyama, H. (2007). The role of ambipolar diffusion in the formation process of moderately magnetized diffuse clouds. Astrophys. J. 658, L99-L102. doi: 10.1086/514816

Inutsuka, S.-i., Machida, M. N., and Matsumoto, T. (2010). Emergence of Protoplanetary Disks and Successive Formation of Gaseous 
Planets by Gravitational Instability. Astrophys. J. Lett. 718, L58-L62. doi: 10.1088/2041-8205/718/2/L58

Joos, M., Hennebelle, P., and Ciardi, A. (2012). Protostellar disk formation and transport of angular momentum during magnetized core collapse. Astron. Astrophys. 543:A128. doi: 10.1051/0004-6361/201118730

Joos, M., Hennebelle, P., Ciardi, A., and Fromang, S. (2013). The influence of turbulence during magnetized core collapse and its consequences on lowmass star formation. Astron. Astrophys. 554:A17. doi: 10.1051/0004-6361/2012 20649

Kataoka, A., Muto, T., Momose, M., Tsukagoshi, T., Fukagawa, M., Shibai, H., et al. (2015). Millimeter-wave polarization of protoplanetary disks due to dust scattering. Astrophys. J. 809:78. doi: 10.1088/0004-637X/809/1/78

Kataoka, A., Tsukagoshi, T., Momose, M., Nagai, H., Muto, T., Dullemond, C. P., et al. (2016). Submillimeter polarization observation of the protoplanetary disk around HD 142527. Astrophys. J. 831:L12. doi: 10.3847/2041-8205/831/2/L12

Kataoka, A., Tsukagoshi, T., Pohl, A., Muto, T., Nagai, H., Stephens, I. W., et al. (2017). The evidence of radio polarization induced by the radiative grain alignment and self-scattering of dust grains in a protoplanetary disk. Astrophys. J. 844:L5. doi: 10.3847/2041-8213/aa7e33

Krasnopolsky, R., Li, Z.-Y., and Shang, H. (2010). Disk formation enabled by enhanced resistivity. Astrophys. J. 716, 1541-1550. doi: 10.1088/0004-637X/716/2/1541

Krasnopolsky, R., Li, Z.-Y., and Shang, H. (2011). Disk formation in magnetized clouds enabled by the Hall effect. Astrophys. J. 733:54. doi: $10.1088 / 0004-637 \mathrm{X} / 733 / 1 / 54$

Krumholz, M. R., Crutcher, R. M., and Hull, C. L. H. (2013). Protostellar disk formation enabled by weak, misaligned magnetic fields. Astrophys. J. 767:L11. doi: 10.1088/2041-8205/767/1/L11

Kunz, M. W., and Mouschovias, T. C. (2009). The nonisothermal stage of magnetic star formation. I. Formulation of the problem and method of solution. Astrophys. J. 693, 1895-1911. doi: 10.1088/0004-637X/693/2/1895

Kunz, M. W., and Mouschovias, T. C. (2010). The non-isothermal stage of magnetic star formation - II. Results. Month. Notices RAS 408, 322-341. doi: $10.1111 /$ j.1365-2966.2010.17110.x

Larson, R. B. (1969). Numerical calculations of the dynamics of collapsing protostar. Month. Notices RAS 145:271. doi: 10.1093/mnras/145.3.271

Lee, C.-F., Li, Z.-Y., Ching, T.-C., Lai, S.-P., and Yang, H. (2018). ALMA dust polarization observations of two young edge-on protostellar disks. Astrophys. J. 854:56. doi: 10.3847/1538-4357/aaa769

Lee, C.-F., Li, Z.-Y., Ho, P. T. P., Hirano, N., Zhang, Q., and Shang, H. (2017). First detection of equatorial dark dust lane in a protostellar disk at submillimeter wavelength. Sci. Adv. 3:e1602935. doi: 10.1126/sciadv.1602935

Lewis, B. T., and Bate, M. R. (2017). The dependence of protostar formation on the geometry and strength of the initial magnetic field. Month. Notices RAS 467, 3324-3337. doi: 10.1093/mnras/stx271

Lewis, B. T., and Bate, M. R. (2018). Shaken and stirred: the effects of turbulence and rotation on disc and outflow formation during the collapse of magnetised molecular cloud cores. Month. Notices RAS. 477, 4241-4256. doi: $10.1093 / \mathrm{mnras} / \mathrm{sty} 829$

Lewis, B. T., Bate, M. R., and Price, D. J. (2015). Smoothed particle magnetohydrodynamic simulations of protostellar outflows with misaligned magnetic field and rotation axes. Month. Notices RAS 451, 288-299. doi: $10.1093 / \mathrm{mnras} / \mathrm{stv} 957$

Li, H.-B., Fang, M., Henning, T., and Kainulainen, J. (2013a). The link between magnetic fields and filamentary clouds: bimodal cloud orientations in the Gould Belt. Month. Notices RAS 436, 3707-3719. doi: 10.1093/mnras/stt1849

Li, Z.-Y., Krasnopolsky, R., and Shang, H. (2011). Non-ideal MHD effects and magnetic braking catastrophe in protostellar disk formation. Astrophys. J. 738:180. doi: $10.1088 / 0004-637 \mathrm{X} / 738 / 2 / 180$

Li, Z.-Y., Krasnopolsky, R., and Shang, H. (2013b). Does magnetic-field-rotation misalignment solve the magnetic braking catastrophe in protostellar disk formation? Astrophys. J. 774:82. doi: 10.1088/0004-637X/774/1/82

Li, Z.-Y., Krasnopolsky, R., Shang, H., and Zhao, B. (2014). On the role of pseudodisk warping and reconnection in protostellar disk formation in turbulent magnetized cores. Astrophys. J. 793:130. doi: $10.1088 / 0004-637 X / 793 / 2 / 130$

Li, Z.-Y., and McKee, C. F. (1996). Hydromagnetic accretion shocks around low-mass protostars. Astrophys. J. 464:373. doi: 10.1086/177329

Li, Z.-Y., and Shu, F. H. (1996). Magnetized singular isothermal toroids. Astrophys. J. 472:211. doi: 10.1086/178056
Liu, H. B., Lai, S.-P., Hasegawa, Y., Hirano, N., Rao, R., Li, I.-H., et al. (2016). Detection of linearly polarized $6.9 \mathrm{~mm}$ continuum emission from the class 0 young stellar object NGC 1333 IRAS4A. Astrophys. J. 821:41. doi: $10.3847 / 0004-637 \mathrm{X} / 821 / 1 / 41$

Mac Low, M.-M., Norman, M. L., Konigl, A., and Wardle, M. (1995). Incorporation of ambipolar diffusion into the ZEUS magnetohydrodynamics code. Astrophys. J. 442, 726-735. doi: 10.1086/175477

Machida, M. N., and Hosokawa, T. (2013). Evolution of protostellar outflow around low-mass protostar. Month. Notices RAS 431, 1719-1744. doi: $10.1093 / \mathrm{mnras} / \mathrm{stt} 291$

Machida, M. N., Inutsuka, S.-i., and Matsumoto, T. (2008). High- and lowvelocity magnetized outflows in the star formation process in a gravitationally collapsing cloud. Astrophys. J. 676, 1088-1108. doi: 10.1086/528364

Machida, M. N., Inutsuka, S.-I., and Matsumoto, T. (2010). Formation process of the circumstellar disk: long-term simulations in the main accretion phase of star formation. Astrophys. J. 724, 1006-1020. doi: 10.1088/0004-637X/724/2/1006

Machida, M. N., Inutsuka, S.-I., and Matsumoto, T. (2011). Effect of magnetic braking on circumstellar disk formation in a strongly magnetized cloud. Publ. ASJ 63:555. doi: 10.1093/pasj/63.3.555

Machida, M. N., Inutsuka, S.-I., and Matsumoto, T. (2014). Conditions for circumstellar disc formation: effects of initial cloud configuration and sink treatment. Month. Notices RAS 438, 2278-2306. doi: 10.1093/mnras/stt2343

Machida, M. N., Matsumoto, T., Hanawa, T., and Tomisaka, K. (2006). Evolution of rotating molecular cloud core with oblique magnetic field. Astrophys. J. 645, 1227-1245. doi: $10.1086 / 504423$

Machida, M. N., Tomisaka, K., and Matsumoto, T. (2004). First MHD simulation of collapse and fragmentation of magnetized molecular cloud cores. Month. Notices RAS 348, L1-L5. doi: 10.1111/j.1365-2966.2004.07402.x

Marchand, P., Masson, J., Chabrier, G., Hennebelle, P., Commerçon, B., and Vaytet, N. (2016). Chemical solver to compute molecule and grain abundances and non-ideal MHD resistivities in prestellar core-collapse calculations. Astron. Astrophys. 592:A18. doi: 10.1051/0004-6361/201526780

Masson, J., Chabrier, G., Hennebelle, P., Vaytet, N., and Commerçon, B. (2016). Ambipolar diffusion in low-mass star formation. I. General comparison with the ideal magnetohydrodynamic case. Astron. Astrophys. 587:A32. doi: 10.1051/0004-6361/201526371

Mathis, J. S., Rumpl, W., and Nordsieck, K. H. (1977). The size distribution of interstellar grains. Astrophys. J. 217, 425-433. doi: 10.1086/ 155591

Matsumoto, T., and Hanawa, T. (2011). Protostellar collapse of magneto-turbulent cloud cores: shape during collapse and outflow formation. Astrophys. J. 728:47. doi: 10.1088/0004-637X/728/1/47

Matsumoto, T., Machida, M. N., and Inutsuka, S.-i. (2017). Circumstellar disks and outflows in turbulent molecular cloud cores: possible formation mechanism for misaligned systems. Astrophys. J. 839:69. doi: 10.3847/1538-4357/aa6a1c

Matsumoto, T., Nakazato, T., and Tomisaka, K. (2006). Alignment of Outflows with Magnetic Fields in Cloud Cores. Astrophys. J. 637, L105-L108. doi: $10.1086 / 500646$

Matsumoto, T., and Tomisaka, K. (2004). Directions of outflows, disks, magnetic fields, and rotation of young stellar objects in collapsing molecular cloud cores. Astrophys. J. 616, 266-282. doi: 10.1086/424897

Maury, A. J., André, P., Hennebelle, P., Motte, F., Stamatellos, D., Bate, M., et al. (2010). Toward understanding the formation of multiple systems. A pilot IRAM-PdBI survey of Class 0 objects. A\&A 512:A40. doi: $10.1051 / 0004-6361 / 200913492$

Maury, A. J., Girart, J. M., Zhang, Q., Hennebelle, P., Keto, E., Rao, R., et al. (2018). Magnetically regulated collapse in the B335 protostar? I. ALMA observations of the polarized dust emission. Month. Notices RAS 477, 27602765. doi: 10.1093/mnras/sty574

McElroy, D., Walsh, C., Markwick, A. J., Cordiner, M. A., Smith, K., and Millar, T. J. (2013). The UMIST database for astrochemistry 2012. Astron. Astrophys. 550:A36. doi: 10.1051/0004-6361/201220465

McKee, C. F., and Ostriker, E. C. (2007). Theory of star formation. Annu. Rev. Astron. Astrophys. 45, 565-687. doi: 10.1146/annurev.astro.45.051806.110602

Mellon, R. R., and Li, Z.-Y. (2008). Magnetic braking and protostellar disk formation: the ideal MHD limit. Astrophys. J. 681, 1356-1376. doi: $10.1086 / 587542$

Mellon, R. R., and Li, Z.-Y. (2009). Magnetic braking and protostellar disk formation: ambipolar diffusion. Astrophys. J. 698, 922-927. doi: 10.1088/0004-637X/698/1/922 
Mestel, L., and Spitzer, Jr., L. (1956). Star formation in magnetic dust clouds. Month. Notices RAS 116:503. doi: 10.1093/mnras/116.5.503

Mouschovias, T. C. (1976). Nonhomologous contraction and equilibria of selfgravitating, magnetic interstellar clouds embedded in an intercloud medium: star formation. II - Results. Astrophys. J. 207, 141-158. doi: 10.1086/154478

Mouschovias, T. C. (1977). A connection between the rate of rotation of interstellar clouds, magnetic fields, ambipolar diffusion, and the periods of binary stars. Astrophys. J. 211, 147-151. doi: 10.1086/154912

Mouschovias, T. C. (1979). Ambipolar diffusion in interstellar clouds - A new solution. Astrophys. J. 228, 475-481. doi: 10.1086/156868

Mouschovias, T. C. (1983). "Magnetic braking and angular momenta of protostars," in Solar and Stellar Magnetic Fields: Origins and Coronal Effects, Volume 102 of IAU Symposium, ed J. O. Stenflo (Dordrecht), 479-484.

Mouschovias, T. C., and Paleologou, E. V. (1979). The angular momentum problem and magnetic braking - an exact time-dependent solution. Astrophys. J. 230, 204-222. doi: 10.1086/157077

Mouschovias, T. C., and Paleologou, E. V. (1980). Magnetic braking of an aligned rotator during star formation - an exact, time-dependent solution. Astrophys. J. 237, 877-899. doi: 10.1086/157936

Mouschovias, T. C., and Spitzer, Jr., L. (1976). Note on the collapse of magnetic interstellar clouds. Astrophys. J. 210:326. doi: 10.1086/154835

Murillo, N. M., Lai, S.-P., Bruderer, S., Harsono, D., and van Dishoeck, E. F. (2013). A Keplerian disk around a Class 0 source: ALMA observations of VLA1623A. Astron. Astrophys. 560:A103. doi: 10.1051/0004-6361/201322537

Myers, A. T., McKee, C. F., Cunningham, A. J., Klein, R. I., and Krumholz, M. R. (2013). The fragmentation of magnetized, massive star-forming cores with radiative feedback. Astrophys. J. 766:97. doi: 10.1088/0004-637X/766/2/97

Nakano, T. and Nakamura, T. (1978). Gravitational instability of magnetized gaseous disks 6. Publ. ASJ 30, 671-680.

Nakano, T., Nishi, R., and Umebayashi, T. (2002). Mechanism of magnetic flux loss in molecular clouds. Astrophys. J. 573, 199-214. doi: 10.1086/340587

Nakano, T., and Umebayashi, T. (1986). Dissipation of magnetic fields in very dense interstellar clouds. I - Formulation and conditions for efficient dissipation. Month. Notices RAS 218, 663-684. doi: 10.1093/mnras/218.4.663

Nishi, R., Nakano, T., and Umebayashi, T. (1991). Magnetic flux loss from interstellar clouds with various grain-size distributions. Astrophys. J. 368, 181194. doi: $10.1086 / 169682$

Oka, T., Geballe, T. R., Goto, M., Usuda, T., and McCall, B. J. (2005). Hot and diffuse clouds near the galactic center probed by metastable $\mathrm{H}^{+}{ }_{3} 1$,. Astrophys. J. 632, 882-893. doi: 10.1086/432679

Okuzumi, S. (2009). Electric charging of dust aggregates and its effect on dust coagulation in protoplanetary disks. Astrophys. J. 698, 1122-1135. doi: 10.1088/0004-637X/698/2/1122

O'Sullivan, S., and Downes, T. P. (2006). An explicit scheme for multifluid magnetohydrodynamics. Month. Notices RAS 366, 1329-1336. doi: 10.1111/j.1365-2966.2005.09898.x

Padoan, P., and Nordlund, A. (2002). The stellar initial mass function from turbulent fragmentation. Astrophys. J. 576, 870-879. doi: 10.1086/341790

Padovani, M., Galli, D., and Glassgold, A. E. (2009). Cosmic-ray ionization of molecular clouds. Astron. Astrophys. 501, 619-631. doi: 10.1051/0004-6361/200911794

Padovani, M., Galli, D., Hennebelle, P., Commerçon, B., and Joos, M. (2014). The role of cosmic rays on magnetic field diffusion and the formation of protostellar discs. Astron. Astrophys. 571:A33. doi: 10.1051/0004-6361/201424035

Planck Collaboration, Ade, P. A. R., Aghanim, N., Alina, D., Alves, M. I. R., Armitage-Caplan, C., Arnaud, M., et al. (2015). Planck intermediate results. XIX. An overview of the polarized thermal emission from Galactic dust. Astron. Astrophys. 576:A104. doi: 10.1051/0004-6361/201424082

Price, D. J., and Bate, M. R. (2007). The impact of magnetic fields on single and binary star formation. Month. Notices RAS 377, 77-90. doi: 10.1111/j.1365-2966.2007.11621.x

Price, D. J., Wurster, J., Tricco, T. S., Nixon, C., Toupin, S., Pettitt, A., et al. (2018). Phantom: a smoothed particle hydrodynamics and magnetohydrodynamics code for astrophysics. Publ. Astron. Soc. Aust. 35:e031. doi: $10.1017 /$ pasa.2018.25

Rao, R., Girart, J. M., Lai, S.-P., and Marrone, D. P. (2014). Detection of a Magnetized Disk around a Very Young Protostar. Astrophys. J. 780:L6. doi: $10.1088 / 2041-8205 / 780 / 1 / \mathrm{L} 6$
Rodgers-Lee, D., Ray, T. P., and Downes, T. P. (2016). Global multifluid simulations of the magnetorotational instability in radially stratified protoplanetary discs. Month. Notices RAS 463, 134-145. doi: $10.1093 / \mathrm{mnras} / \mathrm{stw} 1980$

Sadavoy, S. I., Myers, P. C., Stephens, I. W., Tobin, J., Commerçon, B., Henning, T., et al. (2018). Dust Polarization toward Embedded Protostars in Ophiuchus with ALMA. I. VLA 1623. Astrophys. J. 859:165. doi: 10.3847/1538-4357/aac21a

Saigo, K., and Tomisaka, K. (2006). Evolution of first cores in rotating molecular cores. Astrophys. J. 645, 381-394. doi: 10.1086/504028

Saigo, K., Tomisaka, K., and Matsumoto, T. (2008). Evolution of first cores and formation of stellar cores in rotating molecular cloud cores. Astrophys. J. 674, 997-1014. doi: 10.1086/523888

Sano, T., and Stone, J. M. (2002a). The effect of the Hall term on the nonlinear evolution of the magnetorotational instability. I. Local axisymmetric simulations. Astrophys. J. 570, 314-328. doi: 10.1086/339504

Sano, T., and Stone, J. M. (2002b). The effect of the Hall term on the nonlinear evolution of the magnetorotational instability. II. Saturation level and critical magnetic reynolds number. Astrophys. J. 577, 534-553. doi: 10.1086/342172

Santos-Lima, R., de Gouveia Dal Pino, E. M., and Lazarian, A. (2012). The role of turbulent magnetic reconnection in the formation of rotationally supported protostellar disks. Astrophys. J. 747:21. doi: 10.1088/0004-637X/747/1/21

Santos-Lima, R., de Gouveia Dal Pino, E. M., and Lazarian, A. (2013). Disc formation in turbulent cloud cores: is magnetic flux loss necessary to stop the magnetic braking catastrophe or not? Month. Notices RAS 429, 3371-3378. doi: $10.1093 / \mathrm{mnras} / \mathrm{sts} 597$

Segura-Cox, D. M., Harris, R. J., Tobin, J. J., Looney, L. W., Li, Z.-Y., Chandler, C., et al. (2016). The VLA nascent disk and multiplicity survey: first look at resolved candidate disks around class 0 and I protostars in the perseus molecular cloud. Astrophys. J. 817:L14. doi: 10.3847/2041-8205/817/2/L14

Segura-Cox, D. M., Looney, L. W., Stephens, I. W., Fernández-López, M., Kwon, W., Tobin, J. J., et al. (2015). The magnetic field in the class 0 protostellar disk of L1527. Astrophys. J. 798:L2. doi: 10.1088/2041-8205/798/1/L2

Seifried, D., Banerjee, R., Pudritz, R. E., and Klessen, R. S. (2012). Disc formation in turbulent massive cores: circumventing the magnetic braking catastrophe. Month. Notices RAS 423, L40-L44. doi: 10.1111/j.1745-3933.2012.01253.x

Seifried, D., Banerjee, R., Pudritz, R. E., and Klessen, R. S. (2013). Turbulenceinduced disc formation in strongly magnetized cloud cores. Month. Notices RAS 432, 3320-3331. doi: 10.1093/mnras/stt682

Shu, F. H., Galli, D., Lizano, S., and Cai, M. (2006). Gravitational collapse of magnetized clouds. II. The role of ohmic dissipation. Astrophys. J. 647, 382-389. doi: $10.1086 / 505258$

Spitzer, L. Jr. (1968). Dynamics of Interstellar Matter and the Formation of Stars. Chicago, IL: University of Chicago Press.

Spitzer, L. Jr., and Tomasko, M. G. (1968). Heating of H i regions by energetic particles. Astrophys. J. 152:971. doi: 10.1086/149610

Stephens, I. W., Looney, L. W., Kwon, W., Fernández-López, M., Hughes, A. M., Mundy, L. G., et al. (2014). Spatially resolved magnetic field structure in the disk of a T Tauri star. Nature 514, 597-599. doi: 10.1038/nature13850

Stephens, I. W., Yang, H., Li, Z.-Y., Looney, L. W., Kataoka, A., Kwon, W., et al. (2017). ALMA reveals transition of polarization pattern with wavelength in HL Tau's disk. Astrophys. J. 851:55. doi: 10.3847/1538-4357/ aa998b

Tassis, K., and Mouschovias, T. C. (2005a). Magnetically controlled spasmodic accretion during star formation. I. Formulation of the problem and method of solution. Astrophys. J. 618, 769-782. doi: 10.1086/424479

Tassis, K., and Mouschovias, T. C. (2005b). Magnetically controlled spasmodic accretion during star formation. II. Results. Astrophys. J. 618, 783-794. doi: $10.1086 / 424480$

Tassis, K., and Mouschovias, T. C. (2007a). Protostar formation in magnetic molecular clouds beyond ion detachment. I. Formulation of the problem and method of solution. Astrophys. J. 660, 370-387. doi: 10.1086/ 512760

Tassis, K., and Mouschovias, T. C. (2007b). Protostar formation in magnetic molecular clouds beyond ion detachment. II. Typical axisymmetric solution. Astrophys. J. 660, 388-401. doi: 10.1086/512761

Tassis, K., and Mouschovias, T. C. (2007c). Protostar formation in magnetic molecular clouds beyond ion detachment. III. A parameter study. Astrophys. J. 660, 402-417. doi: $10.1086 / 512762$ 
Tobin, J. J., Hartmann, L., Chiang, H.-F., Wilner, D. J., Looney, L. W., Loinard, L., et al. (2012). A 0.2-solar-mass protostar with a Keplerian disk in the very young L1527 IRS system. Nature 492, 83-85. doi: 10.1038/nature11610

Tomida, K. (2014). Radiation magnetohydrodynamic simulations of protostellar collapse: low-metallicity environments. Astrophys. J. 786:98. doi: 10.1088/0004-637X/786/2/98

Tomida, K., Machida, M. N., Hosokawa, T., Sakurai, Y., and Lin, C. H. (2017). Grand-design spiral arms in a young forming circumstellar disk. Astrophys. J. 835:L11. doi: 10.3847/2041-8213/835/1/L11

Tomida, K., Okuzumi, S., and Machida, M. N. (2015). Radiation magnetohydrodynamic simulations of protostellar collapse: nonideal magnetohydrodynamic effects and early formation of circumstellar disks. Astrophys. J. 801:117. doi: 10.1088/0004-637X/801/2/117

Tomida, K., Tomisaka, K., Matsumoto, T., Hori, Y., Okuzumi, S., Machida, M. N., et al. (2013). Radiation magnetohydrodynamic simulations of protostellar collapse: protostellar core formation. Astrophys. J. 763:6. doi: 10.1088/0004-637X/763/1/6

Tomida, K., Tomisaka, K., Matsumoto, T., Ohsuga, K., Machida, M. N., and Saigo, K. (2010). Radiation magnetohydrodynamics simulation of protostellar collapse: two-component molecular outflow. Astrophys. J. 714, L58-L63. doi: 10.1088/2041-8205/714/1/L58

Tomisaka, K. (2000). The evolution of the angular momentum distribution during star formation. Astrophys. J. 528, L41-L44. doi: 10.1086/312417

Toomre, A. (1964). On the gravitational stability of a disk of stars. Astrophys. J. 139, 1217-1238. doi: 10.1086/147861

Troland, T. H., and Crutcher, R. M. (2008). Magnetic fields in dark cloud cores: arecibo $\mathrm{OH}$ zeeman observations. Astrophys. J. 680, 457-465. doi: 10.1086/587546

Tsukamoto, Y., Iwasaki, K., Okuzumi, S., Machida, M. N., and Inutsuka, S. (2015a). Bimodality of circumstellar disk evolution induced by the hall current. Astrophys. J. 810:L26. doi: 10.1088/2041-8205/810/2/L26

Tsukamoto, Y., Iwasaki, K., Okuzumi, S., Machida, M. N., and Inutsuka, S. (2015b). Effects of Ohmic and ambipolar diffusion on formation and evolution of first cores, protostars, and circumstellar discs. Month. Notices RAS 452, 278-288. doi: $10.1093 / \mathrm{mnras} / \mathrm{stv} 1290$

Tsukamoto, Y., Okuzumi, S., Iwasaki, K., Machida, M. N., and Inutsuka, S.-i. (2017). The impact of the Hall effect during cloud core collapse: implications for circumstellar disk evolution. Publ. ASJ 69:95. doi: 10.1093/pasj/psx113

Turner, N. J., and Sano, T. (2008). Dead zone accretion flows in protostellar disks. Astrophys. J. 679:L131. doi: 10.1086/589540

Umebayashi, T., and Nakano, T. (1981). Fluxes of energetic particles and the ionization rate in very dense interstellar clouds. Publ. ASJ 33:617.

Umebayashi, T., and Nakano, T. (1990). Magnetic flux loss from interstellar clouds. Month. Notices RAS 243, 103-113. doi: $10.1093 / \mathrm{mnras} / 243.1 .103$

Umebayashi, T., and Nakano, T. (2009). Effects of radionuclides on the ionization state of protoplanetary disks and dense cloud cores. Astrophys. J. 690, 69-81. doi: 10.1088/0004-637X/690/1/69

Vaytet, N., Commerçon, B., Masson, J., González, M., and Chabrier, G. (2018). Protostellar birth with ambipolar and ohmic diffusion. Astron. Astrophys. 615:A5. doi: 10.1051/0004-6361/201732075

Wardle, M. (2007). Magnetic fields in protoplanetary disks. Astrophys. Space Sci. 311, 35-45. doi: 10.1007/s10509-007-9575-8

Wardle, M., and Koenigl, A. (1993). The structure of protostellar accretion disks and the origin of bipolar flows. Astrophys. J. 410, 218-238. doi: 10.1086/172739

Wardle, M., and Ng, C. (1999). The conductivity of dense molecular gas. Month. Notices RAS 303, 239-246. doi: 10.1046/j.1365-8711.1999. 02211.x

Ward-Thompson, D., Kirk, J. M., André, P., Saraceno, P., Didelon, P., Könyves, V., et al. (2010). A Herschel study of the properties of starless cores in the Polaris Flare dark cloud region using PACS and SPIRE. Astron. Astrophys. 518:L92. doi: 10.1051/0004-6361/201014618

Weiss, N. O. (1966). The expulsion of magnetic flux by eddies. Proc. R. Soc. Lond. Ser. A 293, 310-328. doi: 10.1098/rspa.1966.0173
Wurster, J. (2016). NICIL: a stand alone library to self-consistently calculate non-ideal magnetohydrodynamic coefficients in molecular cloud cores. Publ. Astron. Soc. Aust. 33:e041. doi: 10.1017/pasa.2016.34

Wurster, J., Bate, M. R., and Price, D. J. (2018a). The collapse of a molecular cloud core to stellar densities using radiation non-ideal magnetohydrodynamics. Month. Notices RAS 475, 1859-1880. doi: 10.1093/mnras/stx3339

Wurster, J., Bate, M. R., and Price, D. J. (2018b). The effect of extreme ionization rates during the initial collapse of a molecular cloud core. Month. Notices RAS 476, 2063-2074. doi: 10.1093/mnras/sty392

Wurster, J., Bate, M. R., and Price, D. J. (2018c). Hall effect-driven formation of gravitationally unstable discs in magnetized molecular cloud cores. Month. Notices RAS 480, 4434-4442. doi: 10.1093/mnras/sty 2212

Wurster, J., Bate, M. R., and Price, D. J. (2018d). On the origin of magnetic fields in stars. Month. Notices RAS 481, 2450-2457. doi: 10.1093/mnras/sty2438

Wurster, J., Price, D. J., and Ayliffe, B. (2014). Ambipolar diffusion in smoothed particle magnetohydrodynamics. Month. Notices RAS 444, 11041112. doi: 10.1093/mnras/stu1524

Wurster, J., Price, D. J., and Bate, M. R. (2016). Can non-ideal magnetohydrodynamics solve the magnetic braking catastrophe? Month. Notices RAS 457, 1037-1061. doi: 10.1093/mnras/stw013

Yang, H., Li, Z.-Y., Looney, L., and Stephens, I. (2016a). Inclination-induced polarization of scattered millimetre radiation from protoplanetary discs: the case of HL Tau. Month. Notices RAS 456, 2794-2805. doi: $10.1093 / \mathrm{mnras} / \mathrm{stv} 2633$

Yang, H., Li, Z.-Y., Looney, L. W., Cox, E. G., Tobin, J., Stephens, I. W., et al. (2016b). Disc polarization from both emission and scattering of magnetically aligned grains: the case of NGC 1333 IRAS 4A1. Month. Notices RAS 460, 4109-4121. doi: 10.1093/mnras/stw1253

Yang, H., Li, Z.-Y., Looney, L. W., Girart, J. M., and Stephens, I. W. (2017). Scattering-produced (sub)millimetre polarization in inclined discs: optical depth effects, near-far side asymmetry and dust settling. Month. Notices RAS 472, 373-388. doi: 10.1093/mnras/stx1951

Yen, H.-W., Koch, P. M., Takakuwa, S., Ho, P. T. P., Ohashi, N., and Tang, Y.W. (2015). Observations of infalling and rotational motions on a 1000 AU scale around 17 class 0 and 0/I protostars: hints of disk growth and magnetic braking? Astrophys. J. 799:193. doi: 10.1088/0004-637X/799/2/193

Yorke, H. W., Bodenheimer, P., and Laughlin, G. (1993). The formation of protostellar disks. I - 1 M(solar). Astrophys. J. 411, 274-284. doi: $10.1086 / 172827$

Yorke, H. W., Bodenheimer, P., and Laughlin, G. (1995). The formation of protostellar disks. 2: disks around intermediate-mass stars. Astrophys. J. 443, 199-208. doi: 10.1086/175514

Zhao, B., Caselli, P., and Li, Z.-Y. (2018). Effect of grain size on differential desorption of volatile species and on non-ideal MHD diffusivity. Month. Notices RAS 478, 2723-2736. doi: 10.1093/mnras/sty1165

Zhao, B., Caselli, P., Li, Z.-Y., Krasnopolsky, R., Shang, H., and Nakamura, F. (2016). Protostellar disc formation enabled by removal of small dust grains. Month. Notices RAS 460, 2050-2076. doi: 10.1093/mnras/stw1124

Zhao, B., Li, Z.-Y., Nakamura, F., Krasnopolsky, R., and Shang, H. (2011). Magnetic flux expulsion in star formation. Astrophys. J. 742:10. doi: $10.1088 / 0004-637 \mathrm{X} / 742 / 1 / 10$

Conflict of Interest Statement: The authors declare that the research was conducted in the absence of any commercial or financial relationships that could be construed as a potential conflict of interest.

Copyright $(9) 2018$ Wurster and Li. This is an open-access article distributed under the terms of the Creative Commons Attribution License (CC BY). The use, distribution or reproduction in other forums is permitted, provided the original author(s) and the copyright owner(s) are credited and that the original publication in this journal is cited, in accordance with accepted academic practice. No use, distribution or reproduction is permitted which does not comply with these terms. 